\section{Induction of muscle-regenerative multipotent stem cells from human adipocytes by PDGF-AB and 5-azacytidine}

\author{
Avani Yeola ${ }^{1,2}$, Shruthi Subramanian ${ }^{1,3}$, Rema A. Oliver ${ }^{4}$, Christine A. Lucas ${ }^{5}$, Julie A. I. Thoms ${ }^{1,2}$, \\ Feng Yan ${ }^{6}$, Jake Olivier ${ }^{7}$, Diego Chacon ${ }^{8}$, Melinda L. Tursky ${ }^{9}$, Pallavi Srivastava ${ }^{10}$, \\ Jason R. Potas ${ }^{11}$, Tzongtyng Hung ${ }^{12}$, Carl Power ${ }^{12}$, Philip Hardy ${ }^{13}$, David D. Ma ${ }^{9}$, \\ Kristopher A. Kilian ${ }^{10}$, Joshua McCarroll ${ }^{14}$, Maria Kavallaris ${ }^{14,15,16}$, Luke B. Hesson ${ }^{3,17}$, Dominik Beck ${ }^{8}$, \\ David J. Curtis ${ }^{6,18}$, Jason W. H. Wong ${ }^{19}$, Edna C. Hardeman ${ }^{5}$, William R. Walsh ${ }^{4}$, Ralph Mobbs ${ }^{4,20}$, \\ Vashe Chandrakanthan $^{1,2 *}$, John E. Pimanda ${ }^{1,2,3,21 *}$
}

Terminally differentiated murine osteocytes and adipocytes can be reprogrammed using platelet-derived growth factor-AB and 5-azacytidine into multipotent stem cells with stromal cell characteristics. We have now optimized culture conditions to reprogram human adipocytes into induced multipotent stem (iMS) cells and characterized their molecular and functional properties. Although the basal transcriptomes of adipocyte-derived iMS cells and adipose tissue-derived mesenchymal stem cells were similar, there were changes in histone modifications and CpG methylation at cis-regulatory regions consistent with an epigenetic landscape that was primed for tissue development and differentiation. In a non-specific tissue injury xenograft model, iMS cells contributed directly to muscle, bone, cartilage, and blood vessels, with no evidence of teratogenic potential. In a cardiotoxin muscle injury model, iMS cells contributed specifically to satellite cells and myofibers without ectopic tissue formation. Together, human adipocyte-derived iMS cells regenerate tissues in a context-dependent manner without ectopic or neoplastic growth.

\section{INTRODUCTION}

The goal of regenerative medicine is to restore function by reconstituting dysfunctional tissues. Most tissues have a reservoir of tissueresident stem cells with restricted cell fates suited to the regeneration of the tissue in which they reside (1-4). The innate regenerative capacity of a tissue is broadly related to the basal rate of tissue turnover, the health of resident stem cells, and the hostility of the local environment. Bone marrow transplants and tissue grafts are frequently used in clinical practice but for most tissues, harvesting and expanding stem and progenitor cells are currently not a viable option $(5,6)$. Given these constraints, research efforts have been focused on converting terminally differentiated cells into pluripotent or lineagerestricted stem cells $(7,8)$. However, tissues are often a complex mix of diverse cell types that are derived from distinct stem cells. Therefore, multipotent stem cells may have advantages over tissue-specific stem cells. To be of use in regenerative medicine, these cells would need to respond appropriately to regional cues and participate in context-dependent tissue regeneration without forming ectopic tis- sues or teratomas. Mesenchymal stem cells (MSCs) were thought to have some of these characteristics (9-11), but despite numerous ongoing clinical trials, evidence for their direct contribution to new tissue formation in humans is sparse, either due to the lack of sufficient means to trace cell fate in hosts in vivo or failure of these cells to regenerate tissues $(12,13)$.

We previously reported a method by which primary terminally differentiated somatic cells could be converted into multipotent stem cells, which we termed as induced multipotent stem (iMS) cells (14). These cells were generated by transiently culturing primary mouse osteocytes in medium supplemented with azacitidine (AZA; 2 days) and platelet-derived growth factor-AB (PDGF-AB; 8 days). Although the precise mechanisms by which these agents promoted cell conversion was unclear, the net effect was reduced DNA methylation at the OCT4 promoter and reexpression of pluripotency factors (OCT4, KLF4, SOX2, c-MYC, SSEA-1, and NANOG) in 2 to $4 \%$ of treated osteocytes. iMS cells resembled MSCs with comparable morphology, cell surface phenotype, colony-forming unit fibroblast (CFU-F),

\footnotetext{
${ }^{1}$ Adult Cancer Program, Lowy Cancer Research Centre, UNSW Sydney, Sydney, NSW 2052, Australia. ${ }^{2}$ School of Medical Sciences, Faculty of Medicine, UNSW Sydney, Sydney, NSW 2052, Australia. ${ }^{3}$ Prince of Wales Clinical School, Faculty of Medicine, UNSW Sydney, Sydney, NSW 2052, Australia. ${ }^{4}$ Surgical and Orthopaedic Research Laboratories, Prince of Wales Clinical School, UNSW Sydney, Sydney, NSW 2052, Australia. ${ }^{5}$ Cellular and Genetic Medicine Unit, School of Medical Sciences, UNSW Sydney, Sydney, NSW 2052, Australia. ${ }^{6}$ Australian Centre for Blood Diseases, Central Clinical School, Monash University, Melbourne, VIC, Australia. ${ }^{7}$ School of Mathematics and Statistics, UNSW Sydney, Sydney, NSW 2052, Australia. ${ }^{8}$ School of Biomedical Engineering, University of Technology Sydney, Sydney, NSW 2007, Australia. ${ }^{9}$ St. Vincent's Centre for Applied Medical Research, St Vincent's Hospital Sydney and St Vincent's Clinical School, Faculty of Medicine, UNSW Sydney, Sydney, NSW 2010, Australia. ${ }^{10}$ School of Material Sciences and Engineering, School of Chemistry, Australian Centre for Nanomedicine, UNSW Sydney, Sydney, NSW 2052, Australia. ${ }^{11}$ Translational Neuroscience Facility, School of Medical Sciences, UNSW Sydney, Sydney, NSW 2052, Australia. ${ }^{12}$ Biological Resources Imaging Laboratory, Mark Wainwright Analytical Centre, UNSW Sydney, Sydney, NSW 2052, Australia. ${ }^{13}$ Cyto Labs, Bentley, WA 6102, Australia. ${ }^{14}$ Children's Cancer Institute, Lowy Cancer Research Centre, University of New South Wales Sydney, Sydney, NSW, Australia. ${ }^{15}$ ARC Centre of Excellence in Convergent Bio-Nano Science and Technology, Australian Centre for Nanomedicine, UNSW Sydney, Sydney, NSW 2052, Australia. ${ }^{16}$ School of Women's and Children's Health, Faculty of Medicine, UNSW Sydney, Sydney, NSW 2052, Australia. ${ }^{17}$ Kinghorn Centre for Clinical Genomics, Garvan Institute of Medical Research, Darlinghurst, NSW 2010, Australia. ${ }^{18}$ Department of Clinical Haematology, Alfred Health, Melbourne, VIC, Australia. ${ }^{19}$ School of Biomedical Sciences, Li Ka Shing Faculty of Medicine, The University of Hong Kong, Pokfulam, Hong Kong Special Administrative Region. ${ }^{20}$ Department of Neurosurgery, Prince of Wales Hospital, Randwick, NSW 2031, Australia. ${ }^{21}$ Department of Haematology, Prince of Wales Hospital, Randwick, NSW 2031, Australia. ${ }^{*}$ Corresponding author. Email: v.chandrakanthan@unsw.edu.au (V.C); jpimanda@unsw.edu.au (J.E.P.)
} 
long-term growth, clonogenicity, and multilineage in vitro differentiation potential. iMS cells also contributed directly to in vivo tissue regeneration and did so in a context-dependent manner without forming teratomas. In proof-of-principle experiments, we also showed that primary mouse and human adipocytes could be converted into long-term repopulating CFU-Fs by this method using a suitably modified protocol (14).

AZA, one of the agents used in this protocol, is a cytidine nucleoside analog and a DNA hypomethylating agent that is routinely used in clinical practice for patients with higher-risk myelodysplastic syndrome (MDS) and for elderly patients with acute myeloid leukemia (AML) who are intolerant to intensive chemotherapy $(15,16)$. AZA is incorporated primarily into RNA, disrupting transcription and protein synthesis. However, 10 to $35 \%$ of drug is incorporated into DNA resulting in the entrapment and depletion of DNA methyltransferases and suppression of DNA methylation (17). Although the relationship between DNA hypomethylation and therapeutic efficacy in MDS/AML is unclear, AZA is known to induce an interferon response and apoptosis in proliferating cells (18-20). PDGF-AB, the other critical reprogramming agent, is one of five PDGF isoforms (PDGF-AA, PDGF-AB, PDGF-BB, PDGF-CC, and PDGF-DD), which bind to one of two PDGF receptors (PDGFR $\alpha$ and PDGFR $\beta$ ) (21). PDGF isoforms are potent mitogens for mesenchymal cells, and recombinant human (rh)PDGF-BB is used as an osteoinductive agent in the clinic (22). PDGF-AB binds preferentially to PDGFR $\alpha$ and induces PDGFR- $\alpha \alpha$ homodimers or PDGFR- $\alpha \beta$ heterodimers. These are activated by autophosphorylation to create docking sites for a variety of downstream signaling molecules (23). Although we have previously demonstrated induction of CFU-Fs from human adipocytes using PDGF-AB/AZA (14), the molecular changes, which underlie conversion, and the multilineage differentiation potential and in vivo regenerative capacity of the converted cells have not been determined.

Here, we report an optimized PDGF-AB/AZA treatment protocol that was used to convert primary human adipocytes, a tissue source that is easily accessible and requires minimal manipulation, from adult donors aged 27 to 66 years into iMS cells with long-term repopulating capacity and multilineage differentiation potential. We also report the molecular landscape of these human iMS cells along with that of MSCs derived from matched adipose tissues and the comparative in vivo regenerative and teratogenic potential of these cells in mouse xenograft models.

\section{RESULTS}

\section{Generating iMS cells from primary human adipocytes}

Primary mature human adipocytes were harvested from subcutaneous fat (Fig. 1A and table S1) and their purity confirmed by flow cytometry with specific attention to the absence of contaminating adiposederived MSCs (AdMSCs) (fig. S1, A and B). As previously described (14), plastic adherent adipocytes were cultured in Alpha Minimum Essential Medium ( $\alpha$ MEM) containing rhPDGF-AB (200 ng/ml) and 20\% autologous serum (AS) with and without $10 \mu \mathrm{M}$ AZA for 2 and 23 days, respectively (Fig. 1A). During daily observations, unilocular lipid globules were observed to fragment within adipocytes $\sim$ day 10 with progressive extrusion of fat into culture medium, coincident with changes in cell morphology (movie S1). Consistent with these observations, when fixed and stained with Oil Red O, adipocytes that were globular in shape at the start of culture resembled lipid laden stromal cells at day 12 and lipid-free stromal cells at day 25 (Fig. 1B).
To evaluate these changes in individual cells, we performed flow cytometry at multiple time points during treatment and probed for adipocyte (LipidTOX) (24) and stromal cell characteristics [PDGFR $\alpha$ expression (25); Fig. 1C]. A subpopulation of adipocytes, when cultured in media supplemented with PDGF-AB/AZA and AS (Fig. 1C, top; treated), showed reduced LipidTOX staining intensity at day 10 , with progressive reduction and complete absence in all cells by day 19. Adipocytes cultured in the absence of PDGF-AB/AZA retained LipidTOX staining, albeit with reduced intensity (Fig. 1C, bottom; untreated). Adipocytes expressed PDGFR $\beta$ [fig. S1C, (i) and (ii)] but not PDGFR $\alpha$ (Fig. 1C) at day 0 but both the frequency and intensity of PDGFR $\alpha$ staining increased from day 21 . To record these changes in real time, we also continuously live-imaged treated adipocytes from days 15 to 25 and recorded the extrusion of fat globules, change in cell morphology from globular to stromal, and acquisition of cell motility and cell mitosis (movie S1 and fig. S1D). Intracellular fragmentation of fat globules was observed over time in untreated adipocytes (fig. $\mathrm{S} 1 \mathrm{E}$ ), consistent with variable LipidTOX staining intensity. CFU-F capacity was absent at day 10 , present in day 15 cultures, and tripled by day 19 with no substantial increase at days 21, 23, and 25 (Fig. 1D). It is noteworthy that CFU-F potential was acquired before PDGFRA surface expression when adipocytes had started to display stromal cell morphology and had diminished fat content. There was also no CFU-F capacity in adipocytes cultured in $\alpha M E M$ with fetal calf serum (FCS) or AS, unless supplemented with both PDGF-AB and AZA. CFU-F capacity was significantly higher with AS than with FCS and absent in serum-free media (SFM) (Fig. 1E and fig. S1F). As previously shown with reprogramming of murine osteocytes, there was dose-dependent inhibition of CFU-F capacity when AG1296, a potent nonselective PDGF receptor tyrosine kinase inhibitor (26), was added to the reprogramming media (Fig. 1F).

To evaluate the impact of patient age and concentrations of PDGF-AB and AZA on the efficiency of human adipocyte conversion, we harvested subcutaneous fat from donors aged $\leq 40(n=3)$, 41 to $60(n=3)$, and $\geq 61(n=3)$ years and subjected each to three different concentrations of PDGF-AB $(100,200$, and $400 \mathrm{ng} / \mathrm{ml})$ and three different concentrations of AZA (5, 10, and $20 \mu \mathrm{M})$ (Fig. 1G). Although all combinations supported cell conversion in all donors across the three age groups, rhPDGF-AB (400 ng/ml) and $5 \mu \mathrm{M} \mathrm{AZA}$ yielded the highest number of CFU-Fs (Fig. 1G). When these cultures were serially passaged in SFM (with no PDGF-AB/AZA supplementation, which was used for cell conversion only), adipocytes converted with reprogramming media containing rhPDGF-AB $(400 \mathrm{ng} / \mathrm{ml})$ and $5 \mu \mathrm{M}$ AZA were sustained the longest (Fig. $1 \mathrm{H}$, fig. S2A, and table S2). The growth plateau that was observed even with these cultures [i.e., adipocytes converted with rhPDGF-AB (400 ng/ml) and $5 \mu \mathrm{M}$ AZA when expanded in SFM or FCS] was overcome when cells were expanded in either autologous or allogeneic human serum (Fig. 1I). The genetic stability of human iMS cells (RM0072 and RM0073) was also assessed using single-nucleotide polymorphism arrays and shown to have a normal copy number profile at a resolution of $250 \mathrm{~kb}$ (fig. S2B). Together, these data identify an optimized protocol for converting human primary adipocytes from donors across different age groups and show that these can be maintained long term in culture.

\section{Molecular and in vitro functional characteristics of human iMS cells}

Given the stromal characteristics observed in human adipocytes treated with PDGF-AB/AZA (Fig. 1), we performed flow cytometry 
A
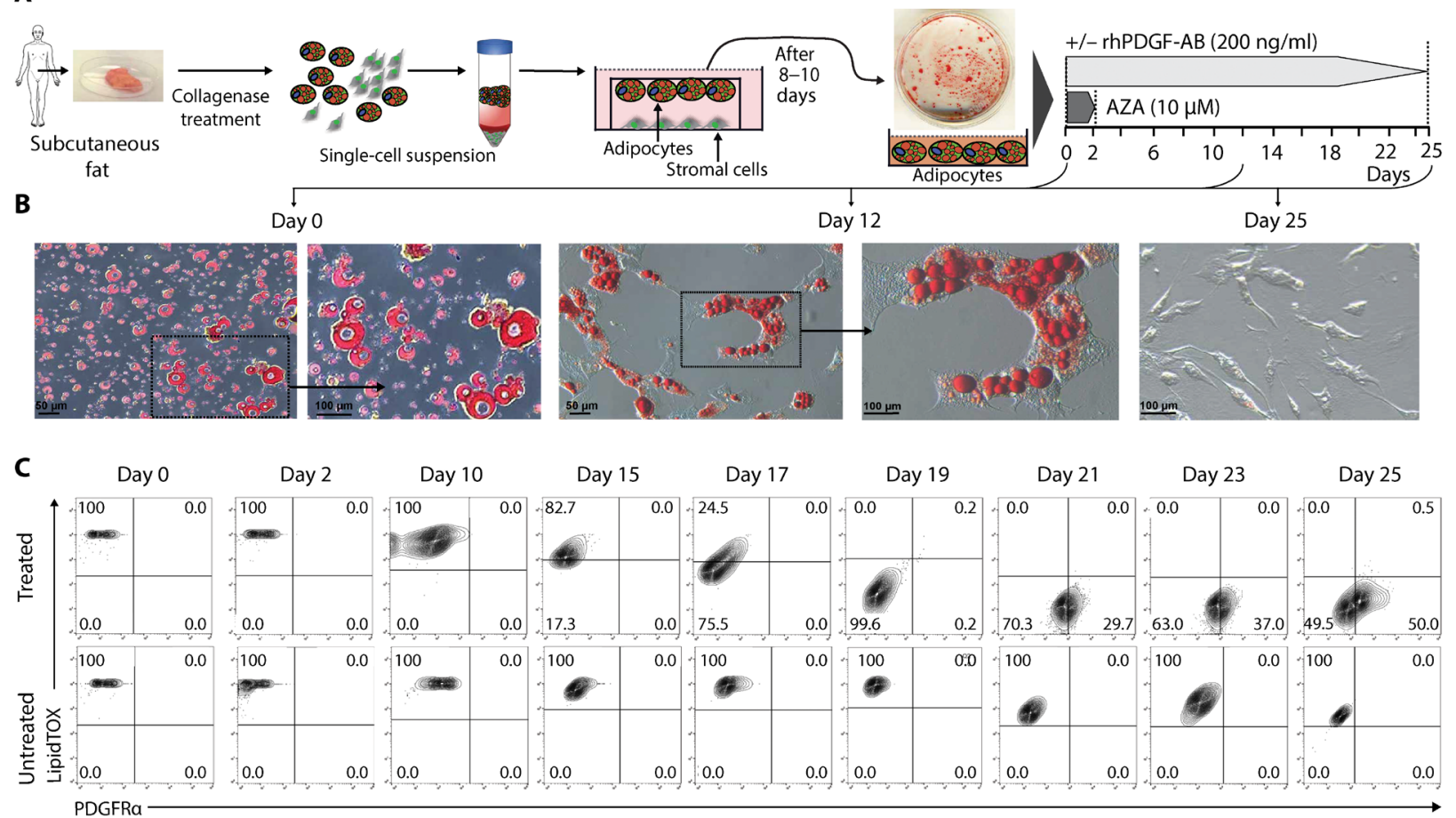

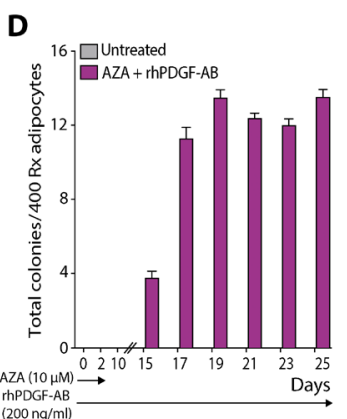

H

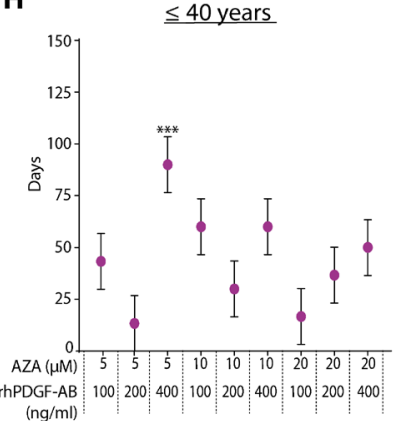

\section{$\mathbf{E}$}
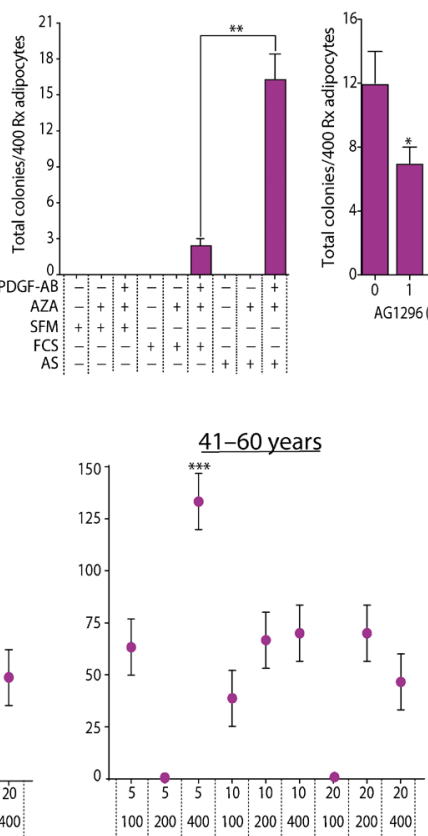

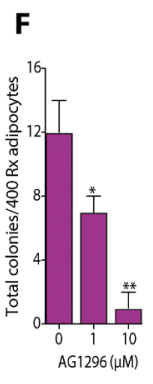

G
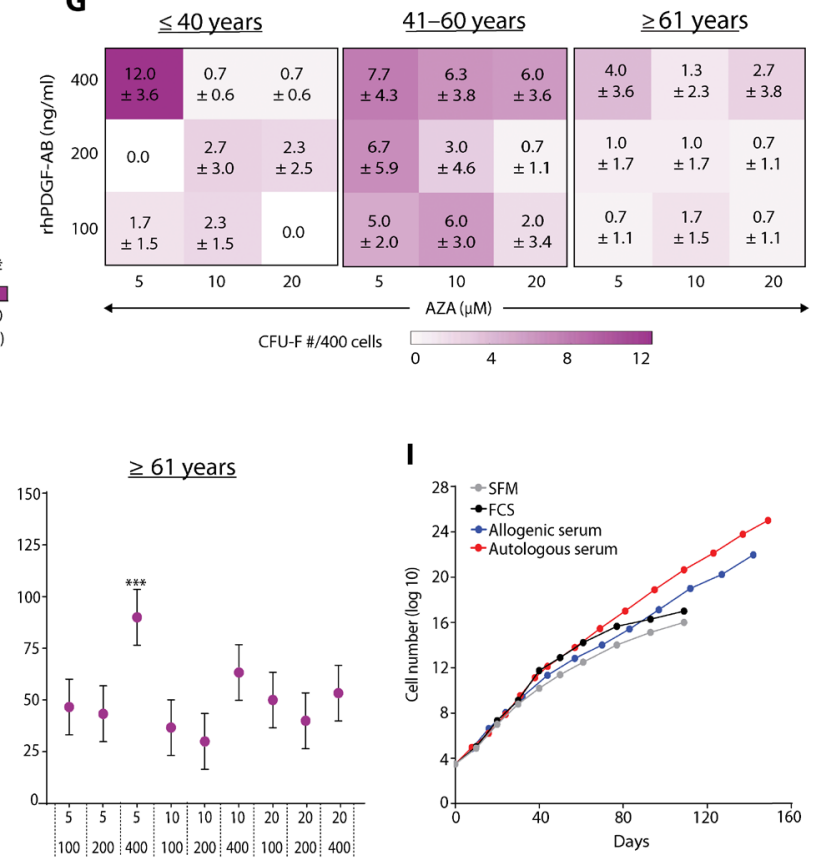

Fig. 1. Generating iMS cells from primary human adipocytes. (A) Generation and reprogramming of adipocytes. (B) Oil Red O-stained adipocytes (days 0 , 12, and 25) during treatment with recombinant human platelet-derived growth factor-AB (rhPDGF-AB) and AZA. (C) Flow cytometry plots of LipidTOX and PDGFR in adipocytes cultured as in (A). (D) CFU-F counts from treated and untreated adipocytes during conversion. (E) CFU-F counts from adipocytes treated (Rx) with indicated combinations of rhPDGF-AB, AZA, fetal calf serum (FCS), autologous serum (AS), or serum-free media (SFM). (F) CFU-F counts from adipocytes reprogrammed in the presence of 0 , 1, or $10 \mu \mathrm{M}$ PDGFR $\alpha / \beta$ inhibitor AG1296. (G) CFU-F counts per 400 reprogrammed adipocytes from three donor age groups $(n=3$ for each) generated using indicated combinations of rhPDGF-AB and AZA. (H) Long-term growth of reprogrammed adipocytes from three donor age groups ( $n=3$ for each) generated using indicated combinations of rhPDGF-AB and AZA. (I) Long-term growth of iMS cells cultured in SFM or media supplemented with FCS, autologous, or allogeneic serum. Error bars indicate SD, $n=3 ;{ }^{*} P<0.05,{ }^{* *} P<0.01$, and ${ }^{* * *} P<0.0001$ calculated using either a Student's $t$ test (E and F) or a linear mixed model (H). Photo credit: Avani Yeola, UNSW Sydney. 
to evaluate their expression of MSC markers CD73, CD90, CD105, and STRO1 (13) and noted expression levels comparable to AdMSCs extracted from the same subcutaneous fat harvest (Fig. 2A). Primary untreated adipocytes (day 25 in culture) did not express any of these MSC markers (fig. S3A). The global transcriptomes of iMS cells and matched AdMSCs were distinct from untreated control adipocytes but were broadly related to each other [Fig. 2B, (i) and (ii)]. Ingenuity pathway analysis (IPA) using genes that were differentially expressed between AdMSCs versus adipocytes [3307 UP/4351 DOWN in AdMSCs versus adipocytes; false discovery rate $(\mathrm{FDR}) \leq 0.05$ ] and iMS versus adipocytes (3311 UP/4400 DOWN in iMS versus adipocytes; FDR $\leq 0.05$ ) showed changes associated with gene expression, posttranslational modification, and cell survival pathways and organismal survival and systems development [Fig. 2B(iii)]. The number of differentially expressed genes between iMS cells and AdMSCs was limited (2 UP/26 DOWN in iMS versus AdMSCs; FDR $\leq 0.05$ ) and too few for confident IPA annotation. All differentially expressed genes and IPA annotations are shown in table S3 (A to E, respectively).

In the absence of significant basal differences in the transcriptomes of AdMSCs and iMS cells, and the use of a hypomethylating agent to induce adipocyte conversion into iMS cells, we examined global enrichment profiles of histone marks associated with transcriptionally active (H3K4me3 and H3K27Ac) and inactive (H3K27me3) chromatin. There were differences in enrichment of specific histone marks in matched AdMSCs versus iMS cells at gene promoters and distal regulatory regions [Fig. 2C(i) and fig. S3, B to D]. H3K4me3, H3K27ac, and $\mathrm{H} 3 \mathrm{~K} 27 \mathrm{me} 3$ enrichments were significantly higher at 255, 107, and 549 regions and significantly lower at 222, 78, and 98 regions in iMS cells versus AdMSCs [Fig. 2C(ii) and table S4, A to C] and were assigned to 237,84 , and 350 and 191, 58, and 67 genes, respectively. IPA was performed using these gene lists to identify biological functions that may be primed in iMS cells relative to AdMSCs [Fig. 2C(iii) and table S4, D to F]. Among these biological functions, annotations for molecular and cellular function (cellular movement, development, growth, and proliferation) and systems development (general; embryonic and tissue development and specific; cardiovascular, skeletal and muscular, and hematological) featured strongly and overlapped across the different epigenetic marks.

We extended these analyses to also assess global CpG methylation in matched AdMSCs and iMS cells using reduced representation bisulfite sequencing [RRBS; (27)]. Again, there were loci with differentially methylated regions (DMRs) in iMS cells versus AdMSCs [Fig. 2D(i)] with increased methylation at 158 and reduced methylation at 397 regions among all regions assessed [Fig. 2D(ii) and table S4G]. IPA of genes associated with these DMRs showed a notable overlap in annotated biological functions [Fig. 2D(iii) and table $\mathrm{S} 4 \mathrm{H}$ ] with those associated with differential $\mathrm{H} 3 \mathrm{~K} 4 \mathrm{me} 3$, H3K27Ac, and H3K27me3 enrichment [Fig. 2C(iii) and table S4, E to G]. Together, these data imply that although basal transcriptomic differences between iMS cells and AdMSCs were limited, there were notable differences in epigenetic profiles at cis-regulatory regions of genes that were associated with cellular growth and systems development.

We next compared iMS cells to adipocytes from which they were derived. Expression of genes associated with adipogenesis was depleted in iMS cells (fig. S4A and table S4I). The promoter regions of these genes in iMS cells had broadly retained an active histone mark (H3K4me3), but, in contrast with adipocytes, many had acquired an inactive mark (H3K27me3) (fig. S4B and table S4J). However, there were examples where iMS cells had lost active histone marks
( $\mathrm{H} 3 \mathrm{~K} 4 \mathrm{me} 3$ and $\mathrm{H} 3 \mathrm{~K} 27 \mathrm{ac}$ ) at gene promoters and potential regulatory regions and gained repressive $\mathrm{H} 3 \mathrm{~K} 27 \mathrm{me} 3$ [e.g., $A D I P O Q$; fig. $\mathrm{S} 4 \mathrm{C}(\mathrm{i})$ ]. In contrast, stromal genes had acquired active histone marks and lost repressive $\mathrm{H} 3 \mathrm{~K} 27 \mathrm{me} 3$ [e.g. EPH2A; fig. S4C(ii)]. It is noteworthy that promoter regions of genes associated with muscle and pericytes (table S4K) were enriched for active histone marks in iMS cells compared with adipocytes [fig. S4D, (i) and (ii)]. We also compared demethylated CpGs in iMS cells and adipocytes (fig. S4E). There were 7366 sites in 2971 genes that were hypomethylated in iMS cells, of which 236 showed increased expression and were enriched for genes associated with tissue development and cellular growth and proliferation (fig. S4E).

PDGF-AB/AZA-treated murine osteocytes (murine iMS cells), but not bone-derived MSCs, expressed pluripotency associated genes, which were detectable by immunohistochemistry in 1 to $4 \%$ of cells (14). To evaluate expression in reprogrammed human cells, PDGF$\mathrm{AB} / \mathrm{AZA}$-treated human adipocytes and matched AdMSCs were stained for OCT4, NANOG, and SOX2 with expression noted in 2, 0.5 , and $3.5 \%$ of iMS cells respectively, but no expression was detected in AdMSCs (Fig. 2E). In addition to these transcription factors, we also evaluated surface expression of TRA-1-60 and SSEA4. Both proteins were uniformly expressed on iPSCs and absent in AdMSCs [fig. S4F(i)] and adipocytes [fig. S4F(ii)]. Although TRA-1-60 was absent in iMS cells, most (78\%) expressed SSEA4 but rarely $(<1 \%)$ coexpressed OCT4 and NANOG [fig. S4F(i)].

MSCs can be induced to differentiate in vitro into various cell lineages in response to specific cytokines and culture conditions. To evaluate the in vitro plasticity of human iMS cells, we induced their differentiation along with matched AdMSCs and primary adipocytes, into bone, fat, and cartilage, as well as into other mesodermal Matrigel tube-forming assays for endothelial cells (CD31) and pericytes (PDGFR $\beta$ ) and muscle (MYH, myosin heavy chain; $\alpha$ SMA, $\alpha$-smooth muscle actin), endodermal (hepatocyte; $\mathrm{HNF} 4 \alpha$, hepatocyte nuclear factor $\alpha$ ), and neuroectodermal (TUJ1; neuron specific class III beta tubulin) lineages (Fig. 2F and fig. S4G). Whereas primary adipocytes remained as such and were resistant to transdifferentiation, iMS cells and AdMSCs showed comparable differentiation potential with the notable exception that only iMS cells generated pericyte-lined endothelial tubes in Matrigel. In keeping with these findings, relative to AdMSCs, iMS cells showed permissive epigenetic marks at pericyte genes [increased H3K4me3 and H3K27Ac; EPHA2 and MCAM; fig. $\mathrm{S} 4 \mathrm{H}(\mathrm{i})$; and reduced $\mathrm{CpG}$ methylation; NOTCH1, SMAD7, TIMP2, AKT1, and VWF; fig. S4H(ii)]. Together with the notable differences in epigenetic profiles, these functional differences and low-level expression of pluripotency genes in iMS cell subsets suggested that these cells could be more amenable than matched AdMSCs to respond to developmental cues in vivo.

\section{In vivo characteristics of human iMS cells}

To evaluate spontaneous teratoma formation and in vivo plasticity of iMS cells, we tagged these cells and their matched AdMSCs with a dual lentiviral reporter, LeGO-iG2-Luc2 (28), that expresses both green fluorescent protein (GFP) and luciferase under the control of the cytomegalovirus promoter (Fig. 3A). To test teratoma-initiating capacity, we implanted tagged cells under the right kidney capsules of NOD Scid Gamma (NSG) mice ( $n=3$ per treatment group) after confirming luciferase/GFP expression in cells in culture (fig. S5, A and B). Weekly bioluminescence imaging (BLI) confirmed retention of cells in situ [Fig. 3B(i)] with progressive reduction in signal 

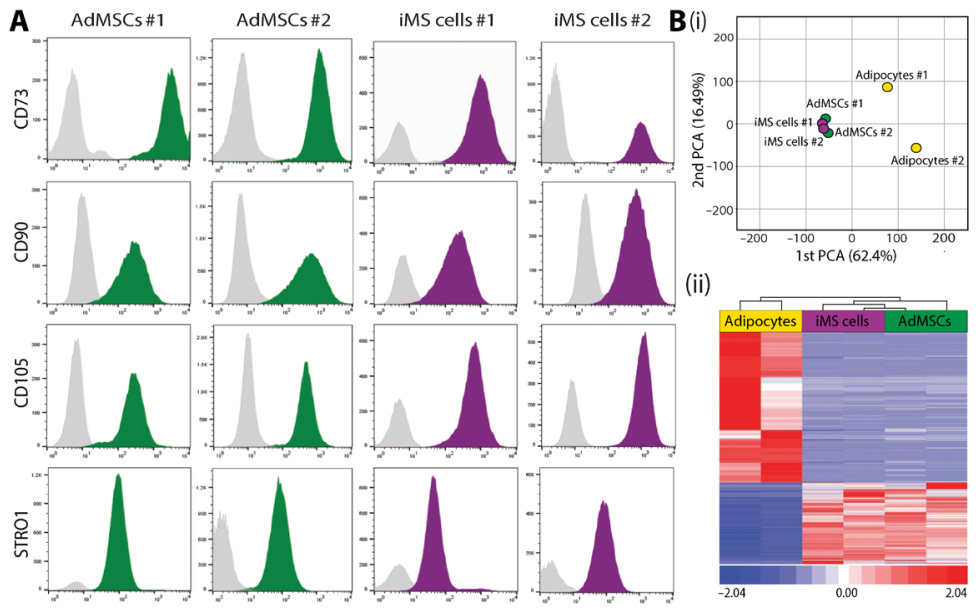

(iii) $\square$ Molecular \& cellular functions $\square$ Systems develooment
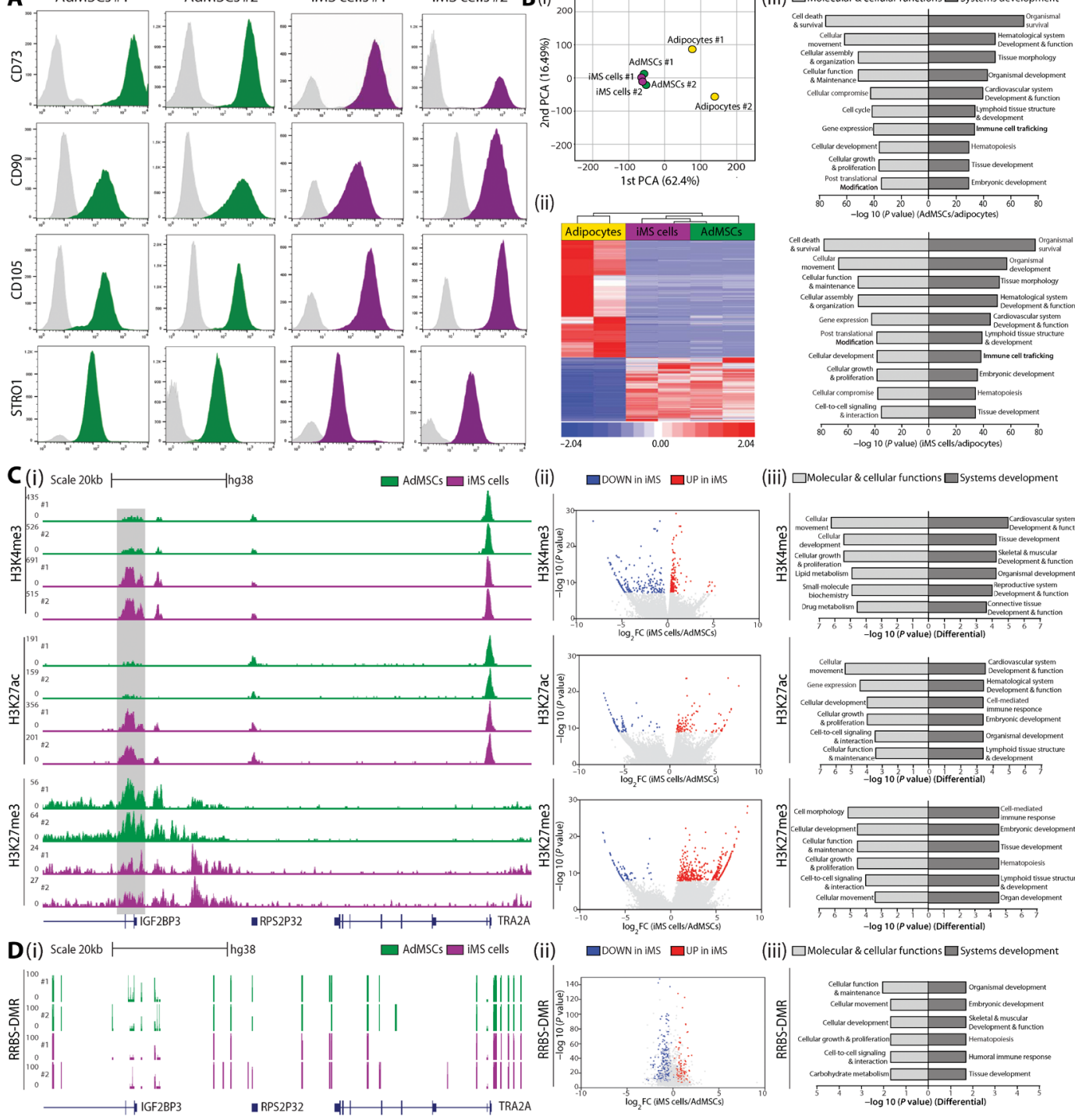

(iii) $\square$ Molecular \& cellular functions $\square$ Systems development

hhg38

$\square$ AdMSCs $\square$ iMs cells
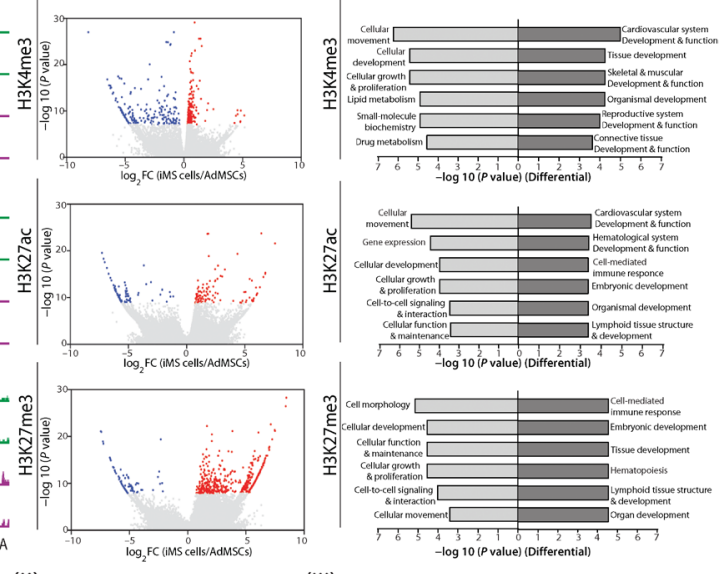

(iii) $\square$ Molecular \& cellular functions $\square$ Systems developmen
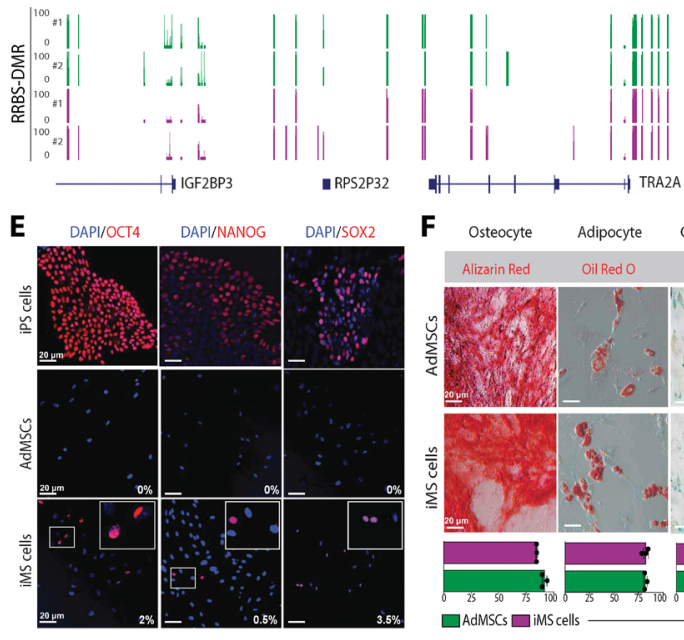

(ii) $\square$ Down in ims $\square$ UP in ims
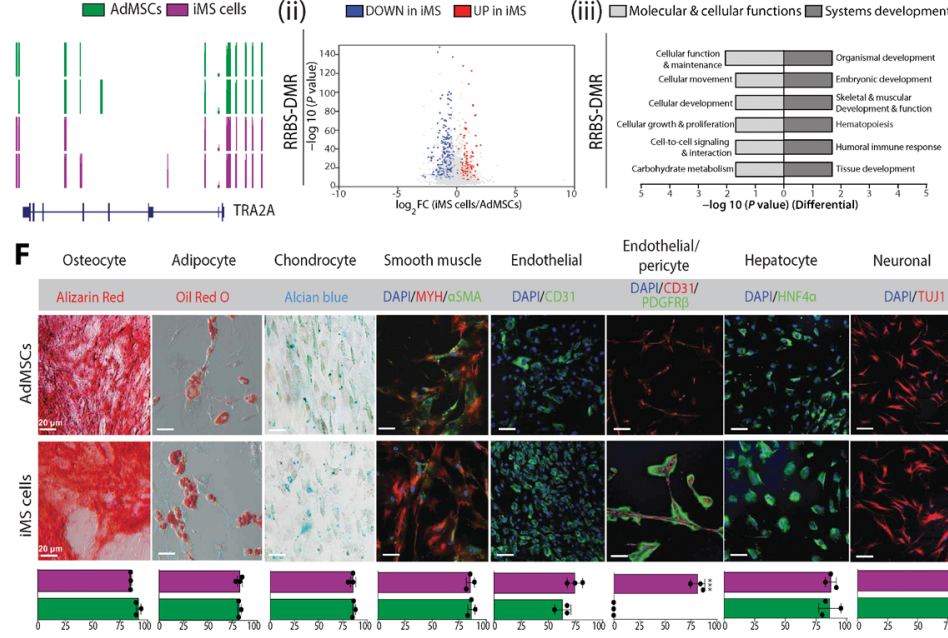

$\square$ AdMSCS $\square$ iMS cells
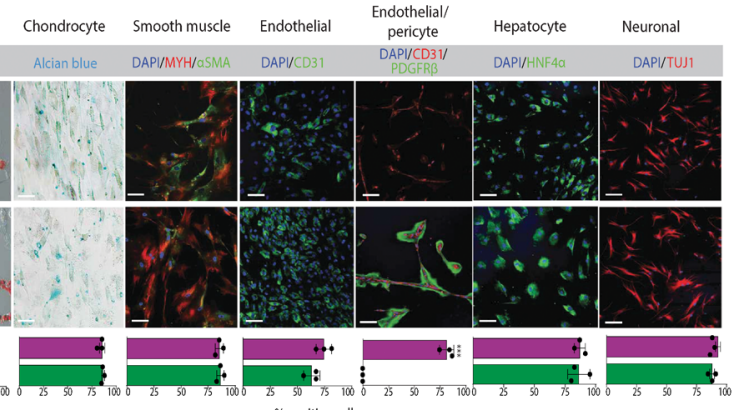

Fig. 2. Molecular and in vitro functional characteristics of human iMS cells. (A) Flow cytometry for stromal markers on AdMSCs (green) and iMS cells (purple) from matched donors. Gray, unstained controls. (B) (i) Principal components analysis (PCA) plot of adipocyte, AdMSC, and iMS transcriptomes. (ii) Hierarchical clustering of differentially expressed genes (DEGs, FDR $\leq 0.05$ ). (iii) Ingenuity pathway analysis (IPA) of DEG between AdMSCs/adipocytes (top) or iMS cells/adipocytes (bottom). The most enriched annotated biological functions are shown. (C) (i) Chromatin immunoprecipitation sequencing (ChIP-seq) profiles in AdMSCs and iMS cells from matched donors at a representative locus. Gray bar indicates differential enrichment. (ii) Volcano plots of H3K4me3, H3K27Ac, and H3K27me3 enrichment peaks significantly UP (red) or DOWN (blue) in iMS cells versus AdMSCs. (iii) IPA of corresponding genes. $\log _{2} \mathrm{FC}$, log2 fold change. (D) (i) DNA methylation at a representative locus in AdMSCs and iMS cells from matched donors. (ii) Volcano plot of regions with significantly higher (red) or lower (blue) DNA methylation in iMS cells versus AdMSCs. (iii) IPA using genes corresponding to differentially methylated regions (DMRs). (E) OCT4, NANOG, and SOX2 expression in iPS, AdMSCs, and iMS cells. Percentage of cells expressing each protein is indicated. DAPI, 4',6-diamidino-2-phenylindole. (F) AdMSCs and iMS cells differentiated in vitro. Bar graphs quantify staining frequencies, error bars show SD, $n=3$. ${ }^{* * *} P<0.001$ (Student's $t$ test). Photo credit: Avani Yeola, UNSW Sydney. 
A
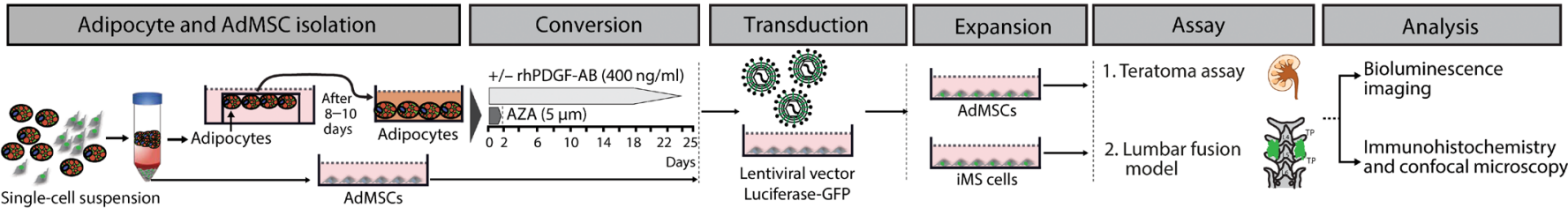

B (i)

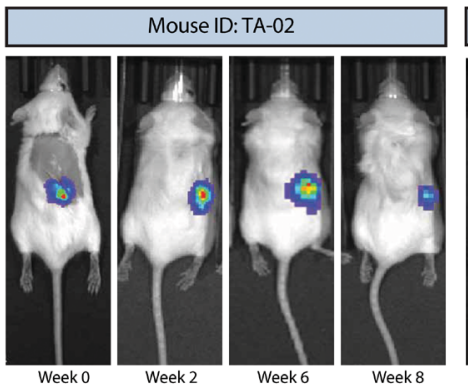

iMS cells

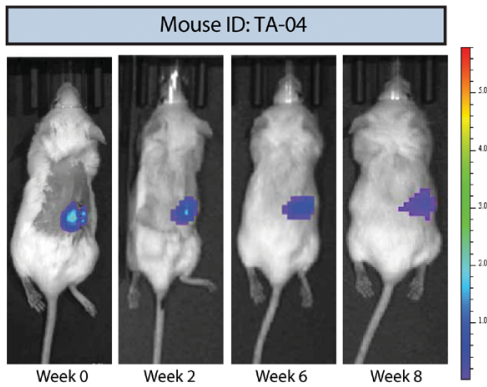

(ii)

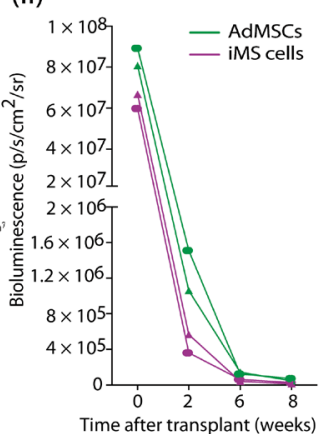

(iii)
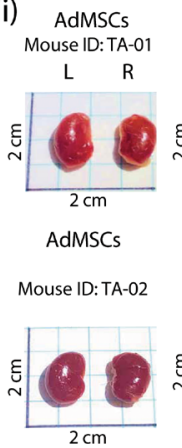

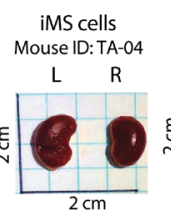

iMS cells

Mouse D: TA-05

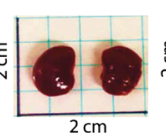

iPS cells Mouse ID: TS-03

$\mathrm{L} \quad \mathrm{R}$

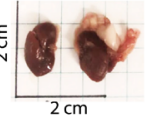

iPS + iMS cells $(1: 49)$ Mouse ID: TS-05

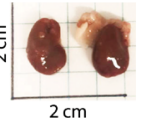

C (i)
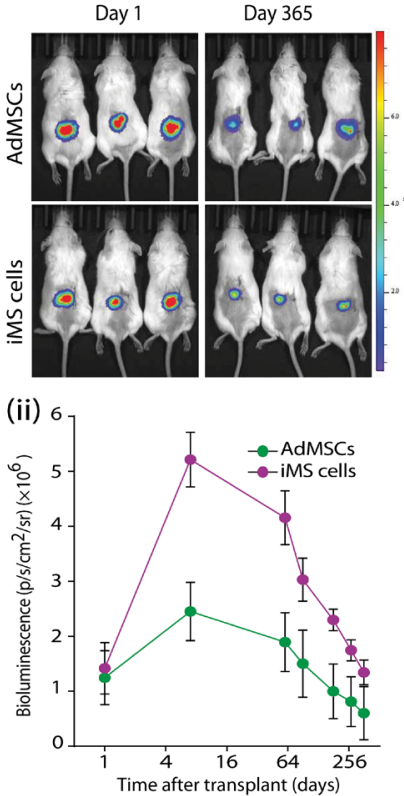

(iii)
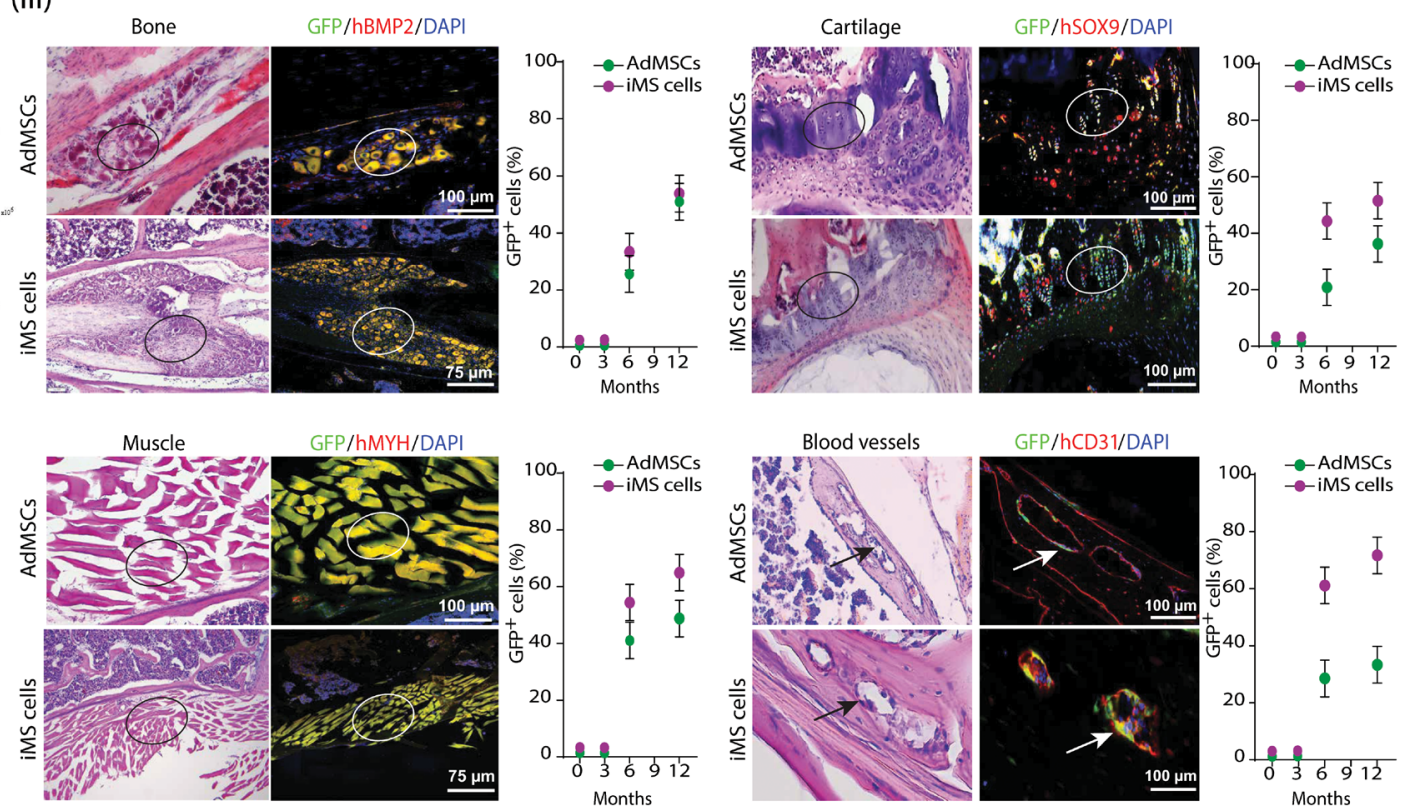

Fig. 3. In vivo characterization of human iMS cells. (A) Generation of luciferase/GFP-reporter AdMSCs and iMS cells, and assessment of their in vivo function. (B) Assessment of teratoma initiating capacity; (i) bioluminescence images at $0,2,6$, and 8 weeks after implantation of $1 \times 10^{6}$ matched AdMSCs and iMS cells (P2; RM0057; $n=2$ per group) under the right kidney capsules. (ii) Quantification of bioluminescence. (iii) Gross kidney morphology 8 weeks following subcapsular implantation of cells (R) or vehicle control (L). (C) Assessment of in vivo plasticity in a posterior-lateral intertransverse lumbar fusion model; (i) bioluminescence images following lumbar implantation of $1 \times 10^{6}$ matched AdMSCs or iMS cells (P2; RM0038; $n=3$ per group) at 1 and 365 days after transplant. (ii) Quantification of bioluminescence. (iii) Tissues (bone, cartilage, muscle, and blood vessels) harvested at 6 months after implantation stained with (left) hematoxylin and eosin or (right) lineage-specific anti-human antibodies circles/ arrows indicate regions covering GFP and lineage marker-positive cells. Corresponding graphs show donor cell (GFP') contributions to bone, cartilage, muscle, and blood vessels as a fraction of total (DAPI ${ }^{+}$) cells in four to five serial tissue sections. Bars indicate confidence interval, $n=3$. Photo Credit: Avani Yeola, UNSW Sydney.

over time [Fig. 3B(ii)] and the absence of teratomas in kidneys injected with either AdMSCs or iMS cells [Fig. 3B(iii)]. Injection of equivalent numbers of iPS cells and iPS + iMS cell mixtures (1:49) to approximate iMS fraction expressing pluripotency markers led to spontaneous tumor formation in the same timeframe [Fig. 3B(iii)].

To evaluate whether iMS cells survived and integrated with damaged tissues in vivo, we implanted transduced human iMS cells and matched AdMSCs controls into a posterior-lateral intertransverse lumbar fusion mouse model (Fig. 3A) (29). Cells were loaded into Helistat collagen sponges 24 hours before implantation into the posterior-lateral gutters adjacent to decorticated lumbar vertebrae of NSG mice ( $n=9$ iMS and $n=9$ AdMSC). Cell retention in situ was confirmed by intraperitoneal injection of D-luciferin $(150 \mathrm{mg} / \mathrm{ml})$ followed by BLI 24 hours after cell implantation, then weekly for the first 6 weeks and monthly up to 12 months from implantation [Fig. 3C(i)]. The BLI signal gradually decreased with time but persisted 
at the site of implantation at 12 months, the final assessment time point [Fig. 3C(ii)]. Groups of mice ( $n=3$ iMS and $n=3$ AdMSC) were euthanized at 3,6, and 12 months and tissues harvested from sites of cell implantation for histology and immunohistochemistry [Fig. 3C(iii)]. Although implanted iMS cells and AdMSCs were present and viable at sites of implantation at 3 months, there was no evidence of lineage-specific gene expression in donor human cells (fig. S5C). By contrast, at 6 months after implantation, $\mathrm{GFP}^{+}$donor iMS cells and AdMSCs were shown to contribute to new bone (BMP2), cartilage (SOX9), muscle (MYH), and endothelium (CD31) at these sites of tissue injury [Fig. 3C(iii)]. The proportion of donor cells expressing lineage-specific markers in a corresponding tissue section was significantly higher in iMS cells compared with matched AdMSCs at 6 months [Fig. 3C(iii) and table S2] as well as 12 months (fig. S5, E and $\mathrm{D}$, and table S2). There was no evidence of malignant growth in any of the tissue sections or evidence of circulating implanted GFP ${ }^{+}$ iMS cells or AdMSCs (fig. S5E). Together, these data show that implanted iMS cells were not teratogenic, were retained long term at sites of implantation, and contributed to regenerating tissues in a contextdependent manner with greater efficiency than matched AdMSCs.

\section{Regenerative potential of human iMS cells in a skeletal muscle injury model}

Although appropriate to assess in vivo plasticity and teratogenicity of implanted cells, the posterior-lateral intertransverse lumber fusion mouse model is not suited to address the question of tissue-specific differentiation and repair in vivo. To this end, we used a muscle injury model (30) where necrosis was induced by injecting $10 \mu \mathrm{M}$ cardiotoxin (CTX) into the left tibialis anterior (TA) muscle of 3-month-old female severe combined immunodeficient (SCID)/ Beige mice. CTX is a myonecrotic agent that spares muscle satellite cells and is amenable to the study of skeletal muscle regeneration. At 24 hours after injury, Matrigel mixed with either $1 \times 10^{6} \mathrm{iMS}$ cells or matched AdMSCs (or no cells as a control) was injected into the damaged TA muscle. The left (injured) and right (uninjured control) TA muscles were harvested at 1,2, or 4 weeks after injury to assess the ability of donor cells to survive and contribute to muscle regeneration without ectopic tissue formation (Fig. 4A; cohort A). Donor human iMS cells or AdMSCs compete with resident murine muscle satellite cells to regenerate muscle, and their regenerative capacity is expected to be handicapped not only by the species barrier but also by having to undergo muscle satellite cell commitment before productive myogenesis. Recognizing this, a cohort of mice was subject to a second CTX injection, 4 weeks from the first injury/cell implantation followed by TA muscle harvest 4 weeks later (Fig. 4A; cohort B).

In tissue sections harvested from cohort $\mathrm{A}$, donor-derived muscle satellite cells (31) [hCD56 (Thermo Fisher Scientific, MA5-11563) ${ }^{+}$; red] were evident in muscles implanted with both iMS cells and AdMSCs at each time point but were most numerous at 2 weeks after implantation [Fig. 4B(i) and fig. S6A]. The frequency of hCD56 cells relative to total satellite cells [sublaminar 4',6-diamidino-2phenylindole-positive $\left(\mathrm{DAPI}^{+}\right)$cells] was quantified in three to five serial sections of TA muscles per mouse in each of three mice per treatment group and was noted to be higher following the implantation of iMS cells compared with AdMSCs at all time points [week $1,5.6 \%$ versus $2.4 \%$; week $2,43.3 \%$ versus $18.2 \%$; and week $4,30.7 \%$ versus 14.6\%; Fig. 4B(i), table S2, and fig. S6A]. Donor cell contribution to regenerating muscle fibers was also assessed by measuring human spectrin (32) costaining with mouse/human laminin [(33) at 4 weeks (Fig. 4B(ii)]. At least 1000 myofibers from three to five serial sections of TA muscles for each of three mice in each treatment group were scored for human $\left[\mathrm{H} ; \mathrm{hSpectrin}^{+}\right.$(full circumference); laminin ${ }^{+}$], murine $\left(\mathrm{M}\right.$; mouse; hSpectrin ${ }^{-}$; laminin $\left.{ }^{+}\right)$, or mouse/ human chimeric [C; hSpectrin ${ }^{+}$(partial circumference); laminin ${ }^{+}$] myofibers. Although none of the myofibers seen in cross section appeared to be completely human (i.e., donor-derived), both iMS cells and AdMSCs contributed to chimeric myofibers [Fig. 4B(ii)]. iMS cell implants contributed to a substantially higher proportion of chimeric fibers than AdMSC implants (57.7\% versus $30.7 \%$; table $\mathrm{S} 2$ ). In cohort B, TA muscles were allowed to regenerate following the initial CTX injection/cell implantation, and re-injured 4 weeks later with a repeat CTX injection. In these mice, although total donor cell contributions to myofibers in TA muscles harvested 4 weeks after re-injury were comparable to that observed in cohort A, there were no myofibers that appeared to be completely human (Fig. 4C). There were substantially more human myofibers following iMS cell implants than with AdMSCs (9.7\% versus 5.4\%; table S2). There was no evidence of ectopic tissue formation in TA muscles following implantation of either iMS cells or AdMSCs in either cohort.

To assess the physiological properties of muscles regenerated with human myofibers, we performed tetanic force contractions in extensor digitorum longus (EDL) muscles following the schema shown in Fig. 4A. Tetanic forces evoked by electrical pulses of various stimulus frequencies were not significantly different between the experimental cohorts or between the experimental cohorts and control animals [fig. S6B, (i) to (iii)]. However, when challenged with a sustained train of electrical pulses [fig. S6C(i)], the iMS group demonstrated significantly greater absolute [fig. S6C(ii)] and specific [fig. S6C(iii)] forces over a 3- to 6-s period. Together, these data showed that iMS cells had the capacity to respond appropriately to the injured environment and contribute to tissue-specific regeneration without impeding function.

\section{DISCUSSION}

We have optimized a protocol, originally designed for mouse osteocytes, to convert human primary adipocytes into iMS cells. We show that these long-term repopulating cells regenerate tissues in vivo in a contextdependent manner without generating ectopic tissues or teratomas.

PDGF-AB, AZA, and serum are indispensable ingredients in reprograming media, but the underlying reasons for their cooperativity and the observed dose-response variability between patients are not known. PDGF-AB is reported to bind and signal via PDGFR- $\alpha \alpha$ and PDGFR- $\alpha \beta$ but not PDGFR- $\beta \beta$ subunits (21). Mouse osteocytes and human adipocytes lack PDGFR $\alpha$, although surface expression was detectable as cells transition during reprogramming [mouse; day 2 of 8 (14) and human day 21 of 25]. However, these cells express PDGFR $\beta$ (14). Given that PDGFR inhibition attenuates iMS cell production in both mice (14) and humans, a degree of facilitated binding of PDGF-AB to PDGF- $\beta \beta$ subunits or signaling through a noncanonical receptor is likely to occur, at least at the start of reprogramming. PDGF-B-containing homo- and heterodimers are potent mitogens that increase the pool of undifferentiated fibroblasts and preosteoblasts with rhPDGF-BB used in the clinic to promote healing of chronic ulcers and bone regeneration (34). However, the unique characteristics of PDGF-AB but not PDGF-BB or PDGF-AA that facilitate reversal and plasticity of cell identity in combination with AZA and serum (14) remain unknown. 
Conversion Expansion

Skeletal muscle injury

Tibialis Anterior (TA):

Cardiotoxin (CTX) injection

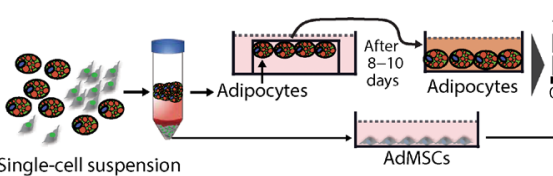

AZA $(5 \mu \mathrm{M})$

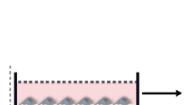

$24 \mathrm{~h}$

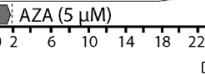

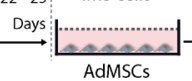

Cohort A

Single-cell suspension

AdMSCs
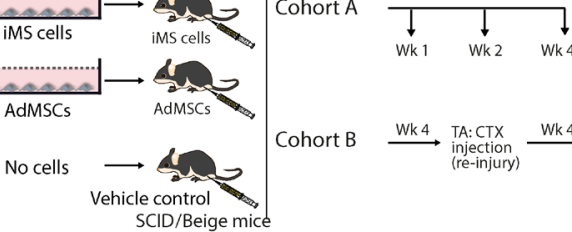

Wk 1 Wk2 Wk4 TA muscle harvest

Confocal

$\stackrel{\text { Wk 4 }}{\longrightarrow} \underset{\text { TA: CTX }}{\text { injection }} \stackrel{\text { Wk4 }}{\longrightarrow}$ TA muscle harvest $\longrightarrow$ imaging

SCID/Beige mice

B

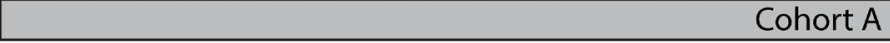

(i)

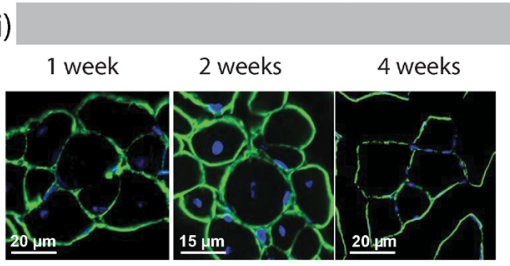

No cells

(ii)
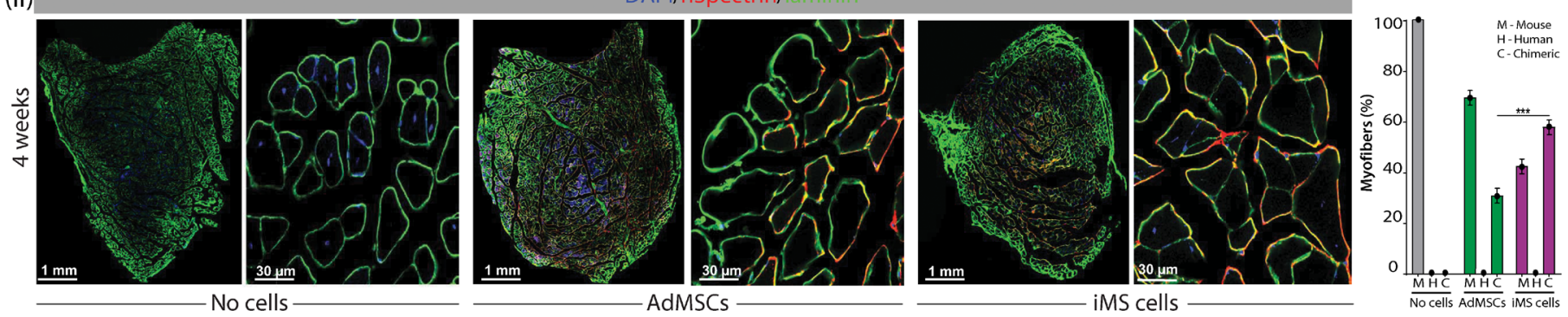

C

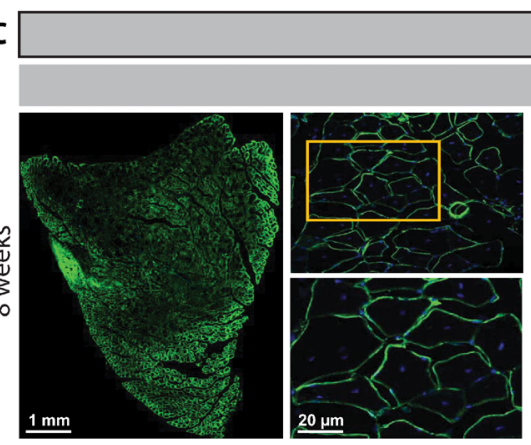

No cells
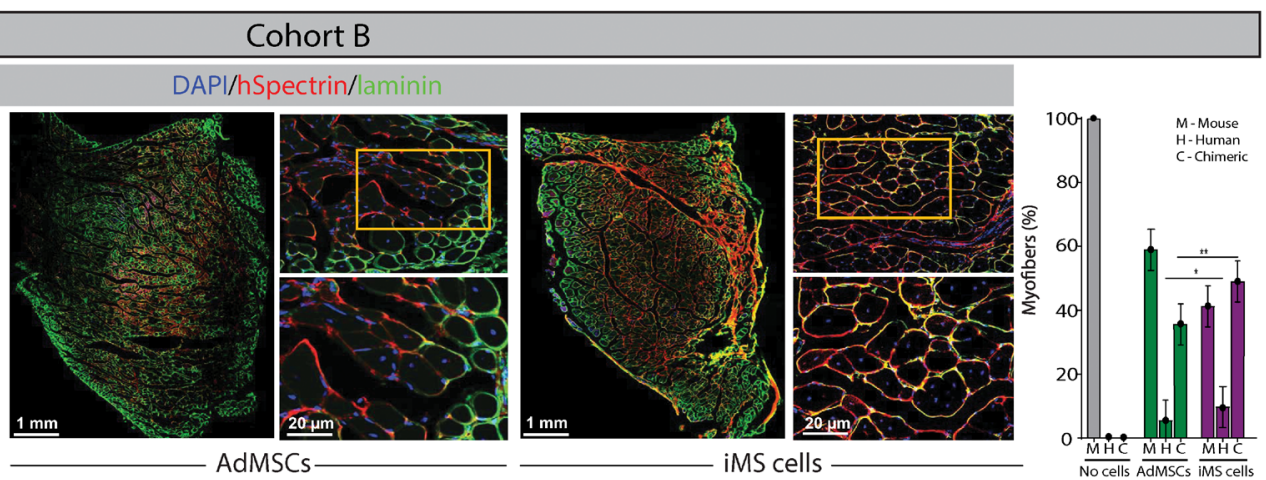

Fig. 4. Regenerative potential of human iMS cells in a skeletal muscle injury model. (A) Generation of iMS and AdMSCs and their assessment in TA muscle injury model. (B) (i) Confocal images of TA muscle stained for human $\mathrm{CD}^{+} 6^{+}$satellite cells (red) and laminin basement membrane protein (green; mouse/human). Graph shows donor hCD56 satellite cell fraction for each treatment group. (ii) Confocal images of TA muscle harvested at 4 weeks and stained for human spectrin (red) and laminin (green; mouse/human). For each treatment, the left panel shows a tile scan of the TA muscle and the right panel a high magnification confocal image. Graph shows contribution of mouse $(\mathrm{M})$, human $(\mathrm{H})$, or chimeric $(\mathrm{C})$ myofibers in three to five serial TA muscle sections per mouse ( $n=3$ mice per treatment group). (C) Confocal images of TA muscle 4 weeks following re-injury with CTX, stained for human spectrin (red) and laminin (green; mouse/human). For each treatment, left panel shows a tile scan of the TA muscle, upper right panel a low-magnification image, and lower right panel a high magnification image of the area boxed above. Graph shows contribution of mouse $(M)$, human $(H)$, or chimeric $(C)$ myofibers in three to five serial TA muscle sections per mouse ( $n=3$ mice per treatment group). Graph bars indicate confidence interval. ${ }^{*} P<0.05,{ }^{* *} P<0.01$, and ${ }^{* *} P<0.001$ (linear mixed model). Photo credit: Avani Yeola, UNSW Sydney.

PDGF-AB was replenished in culture throughout the reprogramming period, but AZA treatment was limited to the first 2 days for both mouse osteocyte and human adipocyte cultures. DNA replication is required for incorporation of AZA into DNA (35) and hence DNA demethylation is unlikely to be an initiating event in the conversion of terminally differentiated nonproliferating cells such as osteocytes and mature adipocytes. However, the majority of intracellular AZA is incorporated into RNA, which could directly affect the cellular transcriptome and proteome as an early event $(36,37)$. It is feasible that subsequent redistribution of AZA from RNA to DNA occurs when cells replicate resulting in DNA hypomethylation as a later event (38). 
In the absence of serum, we could neither convert primary human adipocytes into iMS cells nor perpetuate these cells long term in culture. The efficiency of conversion and expansion was significantly higher with human versus FCS and highest with AS. The precise serum factor(s) that are required for cell conversion in conjunction with PDGF-AB and AZA are not known. The volumes of blood $(\sim 50 \mathrm{ml} \times 2)$ and subcutaneous fat $(5 \mathrm{~g})$ that we harvested from donors were not limiting to generate sufficient numbers of P2 iMS cells $\left(\sim 10 \times 10^{6}\right)$ for in vivo implantation and are in the range of cell numbers used in prospective clinical trials using mesenchymal precursor cells for chronic discogenic lumbar back pain (NCT02412735; $\left.6 \times 10^{6}\right)$ and hypoplastic left heart syndrome (NCT03079401;20 $\times 10^{6}$ ).

Our motivation was to optimize a protocol that could be applied to primary uncultured and easily accessible cells for downstream therapeutic applications, and adipose tissue satisfied these criteria. We have not surveyed other human cell types for their suitability for cell conversion using this protocol. It would be particularly interesting to establish whether tissue-regenerative properties of allogeneic mesenchymal precursor populations that are currently in clinical trials could be boosted by exposure to PDGF-AB/AZA. However, given that iMS cells and MSCs share stromal cell characteristics, identifying a unique set of cell surface markers that can distinguish the former is a priority that would assist in future protocol development and functional assessment of iMS cells.

Producing clinical-grade autologous cells for cell therapy is expensive and challenging requiring suitable quality control measures and certification. However, the advent of chimeric antigen receptor T cell therapy into clinical practice (39) has shown that production of a commercially viable, engineered autologous cellular product is feasible where a need exists. Although there were no apparent genotoxic events in iMS cells at P2, ex vivo expansion of cells could risk accumulation of such events and long-term follow-up of ongoing and recently concluded clinical trials using allogeneic expanded mesenchymal progenitor cells will be instructive with regard to their teratogenic potential. The biological significance of the observed expression of pluripotency-associated transcription factors in 2 to $3 \%$ of murine and human iMS cells is unknown and requires further investigation. However, their presence did not confer teratogenic potential in teratoma assays or at 12-month follow-up despite persistence of cells at the site of implantation. However, this risk cannot be completely discounted, and the clinical indications for iMS or any cell therapy require careful evaluation of need.

In regenerating muscle fibers, it was noteworthy that iMS cells appeared to follow canonical developmental pathways in generating muscle satellite cells that were retained and primed to regenerate muscle following a second muscle-specific injury. Although iMS cells were generated from adipocytes, there was no evidence of any adipose tissue generation. This supports the notion that these cells have lost their native differentiation trajectory and adopted an epigenetic state that favored response to local differentiation cues. The superior in vivo differentiation potential of iMS cells vis-à-vis matched AdMSCs was consistent with our data showing that despite the relatively minor transcriptomic differences between these cell types, the epigenetic state of iMS cells was better primed for systems development. Another clear distinction between iMS cells and AdMSCs was the ability of the former to produce $\mathrm{CD} 31^{+}$endothelial tube-like structures that were enveloped by PDGFR $\beta^{+}$pericytes. An obvious therapeutic application for iMS cells in this context is vascular regeneration in the setting of critical limb ischemia to restore tissue perfusion, an area of clear unmet need (40).
An alternative to ex vivo iMS cell production and expansion is the prospect of in situ reprogramming by local subcutaneous administration of the relevant factors to directly convert subcutaneous adipocytes into iMS cells, thereby eliminating the need for ex vivo cell production. AZA is used in clinical practice and administered as a daily subcutaneous injection for up to 7 days in a 28-day cycle, with responders occasionally remaining on treatment for decades (41). Having determined the optimal dose of AZA required to convert human adipocytes into iMS cells in vitro ( 2 days, $5 \mu \mathrm{M})$, the bridge to ascertaining the comparable in vivo dose would be to first measure levels of AZA incorporation in RNA/DNA following in vitro administration and match the dose of AZA to achieve comparable tissue levels in vivo. A mass spectrometry-based assay was developed to measure in vivo incorporation of AZA metabolites (AZA-MS) in RNA/DNA and is ideally suited to this application (38). The duration of AZA administration for adipocyte conversion was relatively short (i.e., 2 days), but PDGF-AB levels were maintained for 25 days. One mechanism of potentially maintaining local tissue concentrations would be to engineer growth factors to bind extra cellular matrices and be retained at the site of injection. Vascular endothelial growth factor A (VEGF-A) and PDGF-BB have recently been engineered with enhanced syndecan binding and shown to promote tissue healing (42). A comparable approach could help retain PDGF-AB at the site of injection and maintain local concentrations at the required dose. While our current data show that human adipocyte-derived iMS cells regenerate tissues in a context-dependent manner without ectopic or neoplastic growth, these approaches are worth considering as an alternative to an ex vivo expanded cell source in the future.

\section{MATERIALS AND METHODS}

Extended methods for cell growth and differentiation assays and animal models are available in the Supplementary Materials, and antibodies used are detailed in the relevant sections.

\section{Study design}

The primary objective of this study was to optimize conditions that were free of animal products for the generation of human iMS cells from primary adipocytes and to characterize their molecular landscape and function. To this end, we harvested subcutaneous fat from donors across a broad age spectrum and used multiple dose combinations of a recombinant human growth factors and a hypomethylating agent used in the clinic and various serum types. We were particularly keen to demonstrate cell conversion and did so by live imaging and periodic flow cytometry for single-cell quantification of lipid loss and gain of stromal markers. Using our previous report generating mouse iMS cells from osteocytes and adipocytes as a reference, we first characterized the in vitro properties of human iMS cells including (i) long-term growth, (ii) colony-forming potential, (iii) in vitro differentiation, and (iv) molecular landscape. Consistent with their comparative morphology, cell surface markers, and behavioral properties, the transcriptomes (RNA sequencing) were broadly comparable between iMS cells and matched AdMSCs, leading to investigation of epigenetic differences [Assay for TransposaseAccessible Chromatin using sequencing (ATAC-seq) histone chromatin immunoprecipitation sequencing (ChIP-seq), and RRBS for DNA methylation differences] that might explain properties that were unique to iMS cells (expression of pluripotency factors, generation of endothelial tubes in vitro with pericyte envelopes, and in vivo regenerative 
potential). Context-dependent in vivo plasticity was assessed using a tissue injury model that was designed to promote bone/cartilage/ muscle/blood vessel contributions from donor cells and simultaneously assess the absence of ectopic/malignant tissue formation by these cells (labeled and tracked in vivo using a bioluminescence/fluorescence marker). Tissue-specific regeneration and the deployment of canonical developmental pathways were assessed using a specific muscle injury model, and donor cell contributions in all injury models were performed on multiple serial tissue sections in multiple mice with robust statistical analyses (see below). Power calculations were not used, samples were not excluded, and investigators were not blinded. Experiments were repeated multiple times or assessments were performed at multiple time points. Cytogenetic and Copy Number Variation (CNV) analyses were performed on iMS and AdMSCs pretransplant, and their teratogenic potential was assessed both by specific teratoma assays and long-term implantation studies.

\section{Tissue harvest and cell isolation}

Subcutaneous fat and blood were harvested from patients undergoing surgery at the Prince of Wales Hospital, Sydney. Patient tissue was collected in accordance with National Health and Medical Research Council (NHMRC) National Statement on Ethical Conduct in Human Research (2007) and with approval from the South Eastern Sydney Local Health District Human Research Ethics Committee (HREC 14/119). Adipocytes were harvested as described (43). Briefly, adipose tissue was minced and digested with $0.2 \%$ collagenase type 1 (Sigma-Aldrich) at $37^{\circ} \mathrm{C}$ for $40 \mathrm{~min}$ and the homogenized suspension passed through a $70-\mu \mathrm{m}$ filter, inactivated with AS, and centrifuged. Primary adipocytes from the uppermost fatty layer were cultured using the ceiling culture method (44) for 8 to 10 days. AdMSCs from the stromal vascular pellet were cultured in $\alpha \mathrm{MEM}+$ $20 \% \mathrm{AS}+$ penicillin $(100 \mu \mathrm{g} / \mathrm{ml})$ and streptomycin $(250 \mathrm{ng} / \mathrm{ml})$, and $200 \mathrm{mM}$ L-glutamine (complete medium).

Adherent mature adipocytes were cultured in complete medium supplemented with AZA (R\&D systems; 5, 10, and $20 \mu \mathrm{M} ; 2$ days) and rhPDGF-AB (Miltenyi Biotec; 100, 200, and $400 \mathrm{ng} / \mathrm{ml} ; 25$ days) with medium changes every 3 to 4 days. For inhibitor experiments, AG1296 was added for the duration of the culture. Live imaging was performed using an IncuCyte S3 [10× 0.25-numerical aperture (NA) objective] or a Nikon Eclipse Ti-E $(20 \times 0.45$-NA objective). Images were captured every $30 \mathrm{~min}$ for a period of 8 days starting from day 15. Twelve-bit images were acquired with a $1280 \times 1024$ pixel array and analyzed using ImageJ software. In vitro plasticity was determined by inducing the cells to undergo differentiation into various cell types using differentiation protocols adapted from a previous report (45).

\section{Animals}

Animals were housed and bred with approval from the Animal Care and Ethics Committee, University of New South Wales (UNSW; 17/30B, 18/122B, and 18/134B). NSG (NOD.Cg-Prkd ${ }^{\text {scid }} I l 2 r g^{\text {tm } 1 W j l}$ / $\mathrm{SzJ}$ ) and SCID/Beige (C.B-Igh-1b/GbmsTac-Prkdc ${ }^{\text {scid }}-L y s t^{\text {bg }} \mathrm{N}$, sourced from Charles River) strains were used as indicated. The IVIS Spectrum CT (Perkin Elmer) was used to capture bioluminescence. Briefly, 15 min after intraperitoneal injection of D-luciferin $(150 \mathrm{mg} / \mathrm{kg})$, images were acquired for $5 \mathrm{~min}$ and radiance (photon $\mathrm{s}^{-1} \mathrm{~cm}^{-2} \mathrm{sr}^{-1}$ ) was used for subsequent data analysis. The scanned images were analyzed using the Living Image 5.0 software (Perkin Elmer).

\section{Teratoma assay}

Teratoma assays (46) were performed on 3- to 4-month-old female NSG mice. Lentiviral-tagged cells $\left(5 \times 10^{5}\right)$ in $20 \mu$ l of phosphatebuffered saline containing $80 \%$ Matrigel were injected under the right kidney capsule using a fine needle (26 gauges) and followed weekly by BLI until sacrifice at week 8 . Both kidneys were collected, fixed in $4 \%$ paraformaldehyde (PFA) for 48 hours, embedded in optimal cutting temperature compound (OCT), cryosectioned, and imaged for GFP.

\section{Tissue injury models \\ Posterior-lateral intervertebral disc injury model (29)}

Lentiviral-tagged $(28)$ AdMSCs $\left(1 \times 10^{6}\right)$ or iMS cells were loaded onto Helistat collagen sponges and implanted into the postero-lateral gutters in the L4/5 lumbar spine region of anesthetized NSG mice following decortication of the transverse processes. Animals were imaged periodically for bioluminescence to track the presence of transplanted cells. At 3, 6, or 12 months, mice were euthanized, and spines from the thoracic to caudal vertebral region, including the pelvis, were removed whole. The specimens were fixed in $4 \%$ PFA for 48 hours, decalcified in 14\% (w/v) EDTA, and embedded in OCT.

\section{Muscle injury model (47)}

The left TA and EDL muscles of 3- to 4-month-old female SCID/ Beige mice were injured by injection with $15 \mu \mathrm{l}$ of $10 \mu \mathrm{M}$ CTX (Latoxan). Confocal images of three to four serial sections (TA) per mouse were captured by Zen core/AxioVision (Carl Zeiss) and visualized by ImageJ with the colocalization and cell counter plugins [National Institutes of Health; (48)]. Tetanic force contractions were performed on EDL muscles (49).

\section{RNA sequencing}

Total RNA was extracted using the miRNeasy Mini Kit (Qiagen) according to manufacturer's instructions, and 200 ng of total RNA was used for Illumina TruSeq library construction. Library construction and sequencing was performed by Novogene (HK) Co. Ltd. Raw paired-end reads were aligned to the reference genome (hg19) using STAR (https://github.com/alexdobin/STAR), and HTSeq (50) was used to quantify the transcriptomes using the reference "refFlat" database from the UCSC Table Browser (51). The resulting gene expression matrix was normalized and subjected to differential gene expression using DeSeq2 (52). Normalized gene expression was used to compute and plot two-dimensional principal components analysis, using the Python modules sklearn (v0.19.1; https://scikit-learn.org/ stable/) and Matplotlib (v2.2.2; https://matplotlib.org/), respectively. Differentially expressed genes ( $\log _{2}$ fold change $\geq|1|$, adjusted $P<0.05$ ) were the input to produce an unsupervised hierarchical clustering heat map in Partek Genomics Suite software (version 7.0) (Partek Inc., St. Louis, MO, USA). Raw data are available using accession GSE150720.

\section{ChIP sequencing}

ChIP was performed as previously described (53) using antibodies against H3K27Ac (5 $\mu$ g per IP; Abcam, ab4729), H3K4Me3 (5 $\mu$ g per IP; Abcam ab8580), and H3K27Me3 (5 $\mu$ g per IP; Diagenode, C15410195). Library construction and sequencing were performed by Novogene $(\mathrm{HK})$ Co. Ltd. Paired-end reads were aligned to the hg38 genome build using Burrows Wheeler Aligner (BWA) (54) duplicate reads removed using Picard (http://broadinstitute.github.io/picard/), and tracks were generated using DeepTools bamCoverage (https:// deeptools.readthedocs.io/en/develop/). Peaks were called using MACS2 
(55) with the parameter $\left(P=1 \times 10^{-9}\right)$. Differentially bound regions between the AdMSC and iMS were calculated using DiffBind (http:// bioconductor.org/packages/release/bioc/vignettes/DiffBind/inst/ doc/DiffBind.pdf) and regions annotated using ChIPseeker (56). Raw data are available using accession GSE151527. Adipocyte ChIP data were downloaded from Gene Expression Omnibus (GEO); accession numbers are as follows for the three histone marks: GSM916066, GSM670041, and GSM772771.

\section{Reduced representation bisulfite sequencing}

Total genomic DNA was extracted using the DNA MiniPrep Kit (Qiagen), and RRBS library construction and sequencing were performed by Novogene (HK) Co. Ltd. Raw RRBS data in fastq format were quality and adapter trimmed using trim_galore (0.6.4) with -rrbs parameter (www.bioinformatics.babraham.ac.uk/projects/trim_galore). The trimmed fastq files were then aligned to a bisulfite-converted genome (Ensembl GRCh38) using Bismark (2.3.5), and methylation status at each CpG loci was extracted (57). The cytosine coverage files were converted to BigWig format for visualization. Differentially methylated cytosines (DMCs) and DMRs were identified using methylKit (1.10) and edmr (0.6.4.1) packages in $\mathrm{R}(3.6 .1)(58,59)$. DMCs and DMRs were annotated using ChIPseeker (56), and pathway enrichment was performed as detailed below. Raw data are available using accession number GSE151527. Adipocyte RRBS data were downloaded from GEO: GSM2342293 and GSM2342392.

\section{Pathway analysis}

IPA (Qiagen) was used to investigate enrichment in molecular and cellular functions, systems development and function, and canonical pathways.

\section{Statistical analysis}

Statistical analysis was performed in SAS. For the dose-optimization experiments (Fig. 1), a linear mixed model with participant-level random effects was used to estimate maximum time by dose level and age group. A linear mixed model with participant-level random effects was used to analyze statistical differences in lineage contribution outcomes between treatment groups (Fig. 3) and at different time points posttransplant, to estimate the percentage of cells by treatment and lineage. For the in vivo regeneration experiment (Fig. 4), a linear model was used to model the percent of cells over time for each group. Quadratic time terms were added to account for the observed increase from 1 to 2 weeks and decrease from 2 to 4 weeks. In the muscle regeneration experiment, a linear model was applied to cohort $\mathrm{A}$ and cohort $\mathrm{B}$, to estimate and compare percent cells by treatment and source. Statistical modeling data are included in table S2.

\section{SUPPLEMENTARY MATERIALS}

Supplementary material for this article is available at http://advances.sciencemag.org/cgi/ content/full/7/3/eabd1929/DC1

View/request a protocol for this paper from Bio-protocol.

\section{REFERENCES AND NOTES}

1. H. Xia, X. Li, W. Gao, X. Fu, R. H. Fang, L. Zhang, K. Zhang, Tissue repair and regeneration with endogenous stem cells. Nature Reviews Materials 3, 174-193 (2018).

2. A. S. Brack, T. A. Rando, Tissue-specific stem cells: Lessons from the skeletal muscle satellite cell. Cell Stem Cell 10, 504-514 (2012).

3. N. Barker, S. Bartfeld, H. Clevers, Tissue-resident adult stem cell populations of rapidly self-renewing organs. Cell Stem Cell 7, 656-670 (2010).
4. C. Blanpain, E. Fuchs, Stem cell plasticity. Plasticity of epithelial stem cells in tissue regeneration. Science 344, 1242281 (2014).

5. T. R. Heathman, A. W. Nienow, M. J. McCall, K. Coopman, B. Kara, C. J. Hewitt, The translation of cell-based therapies: Clinical landscape and manufacturing challenges. Regen. Med. 10, 49-64 (2015).

6. S. Dimmeler, S. Ding, T. A. Rando, A. Trounson, Translational strategies and challenges in regenerative medicine. Nat. Med. 20, 814-821 (2014).

7. Y. Shi, H. Inoue, J. C. Wu, S. Yamanaka, Induced pluripotent stem cell technology: A decade of progress. Nat. Rev. Drug Discov. 16, 115-130 (2017).

8. C. Jopling, S. Boue, J. C. Izpisua Belmonte, Dedifferentiation, transdifferentiation and reprogramming: Three routes to regeneration. Nat. Rev. Mol. Cell Biol. 12, 79-89 (2011).

9. M. F. Pittenger, D. E. Discher, B. M. Peault, D. G. Phinney, J. M. Hare, A. I. Caplan, Mesenchymal stem cell perspective: Cell biology to clinical progress. NPJ Regen Med 4, 22 (2019).

10. P. Bianco, X. Cao, P. S. Frenette, J. J. Mao, P. G. Robey, P. J. Simmons, C. Y. Wang, The meaning, the sense and the significance: Translating the science of mesenchymal stem cells into medicine. Nat. Med. 19, 35-42 (2013).

11. K. English, A. French, K. J. Wood, Mesenchymal stromal cells: Facilitators of successful transplantation? Cell Stem Cell 7, 431-442 (2010).

12. H. Caplan, S. D. Olson, A. Kumar, M. George, K. S. Prabhakara, P. Wenzel, S. Bedi, N. E. Toledano-Furman, F. Triolo, J. Kamhieh-Milz, G. Moll, C. S. Cox Jr., Mesenchymal stromal cell therapeutic delivery: Translational challenges to clinical application. Front. Immunol. 10, 1645 (2019).

13. M. Dominici, K. Le Blanc, I. Mueller, I. Slaper-Cortenbach, F. Marini, D. Krause, R. Deans, A. Keating, D. Prockop, E. Horwitz, Minimal criteria for defining multipotent mesenchymal stromal cells. The International Society for Cellular Therapy position statement. Cytotherapy 8, 315-317 (2006).

14. V. Chandrakanthan, A. Yeola, J. C. Kwan, R. A. Oliver, Q. Qiao, Y. C. Kang, P. Zarzour, D. Beck, L. Boelen, A. Unnikrishnan, J. E. Villanueva, A. C. Nunez, K. Knezevic, C. Palu, R. Nasrallah, M. Carnell, A. Macmillan, R. Whan, Y. Yu, P. Hardy, S. T. Grey, A. Gladbach, F. Delerue, L. Ittner, R. Mobbs, C. R. Walkley, L. E. Purton, R. L. Ward, J. W. Wong, L. B. Hesson, W. Walsh, J. E. Pimanda, PDGF-AB and 5-Azacytidine induce conversion of somatic cells into tissue-regenerative multipotent stem cells. Proc. Natl. Acad. Sci. U.S.A. 113, E2306-E2315 (2016).

15. L. R. Silverman, D. R. McKenzie, B. L. Peterson, J. F. Holland, J. T. Backstrom, C. L. Beach, R. A. Larson; Cancer, B. Leukemia Group, Further analysis of trials with azacitidine in patients with myelodysplastic syndrome: Studies 8421,8921 , and 9221 by the Cancer and Leukemia Group B. J. Clin. Oncol. 24, 3895-3903 (2006).

16. H. Dombret, J. F. Seymour, A. Butrym, A. Wierzbowska, D. Selleslag, J. H. Jang, R. Kumar, J. Cavenagh, A. C. Schuh, A. Candoni, C. Recher, I. Sandhu, T. Bernal del Castillo, H. K. Al-Ali, G. Martinelli, J. Falantes, R. Noppeney, R. M. Stone, M. D. Minden, H. McIntyre, S. Songer, L. M. Lucy, C. L. Beach, H. Dohner, International phase 3 study of azacitidine vs conventional care regimens in older patients with newly diagnosed AML with $>30 \%$ blasts. Blood 126, 291-299 (2015).

17. J. Diesch, A. Zwick, A. K. Garz, A. Palau, M. Buschbeck, K. S. Gotze, A clinical-molecular update on azanucleoside-based therapy for the treatment of hematologic cancers. Clin. Epigenetics 8, 71 (2016).

18. K. B. Chiappinelli, P. L. Strissel, A. Desrichard, H. Li, C. Henke, B. Akman, A. Hein, N. S. Rote, L. M. Cope, A. Snyder, V. Makarov, S. Budhu, D. J. Slamon, J. D. Wolchok, D. M. Pardoll, M. W. Beckmann, C. A. Zahnow, T. Merghoub, T. A. Chan, S. B. Baylin, R. Strick, Inhibiting DNA methylation causes an interferon response in cancer via dsRNA including endogenous retroviruses. Cell 162, 974-986 (2015).

19. D. Roulois, H. Loo Yau, R. Singhania, Y. Wang, A. Danesh, S. Y. Shen, H. Han, G. Liang, P. A. Jones, T. J. Pugh, C. O'Brien, D. D. De Carvalho, DNA-demethylating agents target colorectal cancer cells by inducing viral mimicry by endogenous transcripts. Cell 162, 961-973 (2015).

20. A. Unnikrishnan, E. Papaemmanuil, D. Beck, N. P. Deshpande, A. Verma, A. Kumari, P. S. Woll, L. A. Richards, K. Knezevic, V. Chandrakanthan, J. A. I. Thoms, M. L. Tursky, Y. Huang, Z. Ali, J. Olivier, S. Galbraith, A. G. Kulasekararaj, M. Tobiasson, M. Karimi, A. Pellagatti, S. R. Wilson, R. Lindeman, B. Young, R. Ramakrishna, C. Arthur, R. Stark, P. Crispin, J. Curnow, P. Warburton, F. Roncolato, J. Boultwood, K. Lynch, S. E. W. Jacobsen, G. J. Mufti, E. Hellstrom-Lindberg, M. R. Wilkins, K. L. MacKenzie, J. W. H. Wong, P. J. Campbell, J. E. Pimanda, Integrative genomics identifies the molecular basis of resistance to azacitidine therapy in myelodysplastic syndromes. Cell Rep. 20, 572-585 (2017).

21. C. H. Heldin, J. Lennartsson, B. Westermark, Involvement of platelet-derived growth factor ligands and receptors in tumorigenesis. J. Intern. Med. 283, 16-44 (2018).

22. F. Li, F. Yu, X. Xu, C. Li, D. Huang, X. Zhou, L. Ye, L. Zheng, Evaluation of recombinant human FGF-2 and PDGF-BB in periodontal regeneration: A systematic review and meta-analysis. Sci. Rep. 7, 65 (2017). 
23. H. Porsch, M. Mehic, B. Olofsson, P. Heldin, C. H. Heldin, Platelet-derived growth factor $\beta$-receptor, transforming growth factor $\beta$ type I receptor, and CD44 protein modulate each other's signaling and stability. J. Biol. Chem. 289, 19747-19757 (2014).

24. S. M. Majka, H. L. Miller, K. M. Helm, A. S. Acosta, C. R. Childs, R. Kong, D. J. Klemm, Analysis and isolation of adipocytes by flow cytometry. Methods Enzymol. 537, 281-296 (2014).

25. J. Andrae, R. Gallini, C. Betsholtz, Role of platelet-derived growth factors in physiology and medicine. Genes Dev. 22, 1276-1312 (2008).

26. M. Kovalenko, A. Gazit, A. Bohmer, C. Rorsman, L. Ronnstrand, C. H. Heldin J. Waltenberger, F. D. Bohmer, A. Levitzki, Selective platelet-derived growth factor receptor kinase blockers reverse sis-transformation. Cancer Res. 54, 6106-6114 (1994).

27. A. Meissner, A. Gnirke, G. W. Bell, B. Ramsahoye, E. S. Lander, R. Jaenisch, Reduced representation bisulfite sequencing for comparative high-resolution DNA methylation analysis. Nucleic Acids Res. 33, 5868-5877 (2005).

28. K. Weber, U. Bartsch, C. Stocking, B. Fehse, A multicolor panel of novel lentiviral "gene ontology" (LeGO) vectors for functional gene analysis. Mol. Ther. 16, 698-706 (2008).

29. R. D. Rao, V. B. Bagaria, B. C. Cooley, Posterolateral intertransverse lumbar fusion in a mouse model: Surgical anatomy and operative technique. Spine J. 7, 61-67 (2007).

30. B. Sacchetti, A. Funari, C. Remoli, G. Giannicola, G. Kogler, S. Liedtke, G. Cossu, M. Serafini, M. Sampaolesi, E. Tagliafico, E. Tenedini, I. Saggio, P. G. Robey, M. Riminucci, P. Bianco, No identical "mesenchymal stem cells" at different times and sites: Human committed progenitors of distinct origin and differentiation potential are incorporated as adventitial cells in microvessels. Stem Cell Reports 6, 897-913 (2016).

31. W. Schubert, K. Zimmermann, M. Cramer, A. Starzinski-Powitz, Lymphocyte antigen Leu-19 as a molecular marker of regeneration in human skeletal muscle. Proc. Natl. Acad. Sci. U.S.A. 86, 307-311 (1989).

32. S. T. Appleyard, M. J. Dunn, V. Dubowitz, M. L. Scott, S. J. Pittman, D. M. Shotton, Monoclonal antibodies detect a spectrin-like protein in normal and dystrophic human skeletal muscle. Proc. Natl. Acad. Sci. U.S.A. 81, 776-780 (1984).

33. T. Sasaki, R. Fassler, E. Hohenester, Laminin: The crux of basement membrane assembly. J. Cell Biol. 164, 959-963 (2004)

34. G. E. Friedlaender, S. Lin, L. A. Solchaga, L. B. Snel, S. E. Lynch, The role of recombinant human platelet-derived growth factor-BB (rhPDGF-BB) in orthopaedic bone repair and regeneration. Curr. Pharm. Des. 19, 3384-3390 (2013).

35. C. Stresemann, F. Lyko, Modes of action of the DNA methyltransferase inhibitors azacytidine and decitabine. Int. J. Cancer 123, 8-13 (2008).

36. L. H. Li, E. J. Olin, H. H. Buskirk, L. M. Reineke, Cytotoxicity and mode of action of 5-azacytidine on L1210 leukemia. Cancer Res. 30, 2760-2769 (1970).

37. J. Aimiuwu, H. Wang, P. Chen, Z. Xie, J. Wang, S. Liu, R. Klisovic, A. Mims, W. Blum, G. Marcucci, K. K. Chan, RNA-dependent inhibition of ribonucleotide reductase is a major pathway for 5-azacytidine activity in acute myeloid leukemia. Blood 119, 5229-5238 (2012)

38. A. Unnikrishnan, A. N. Q. Vo, R. Pickford, M. J. Raftery, A. C. Nunez, A. Verma, L. B. Hesson, J. E. Pimanda, AZA-MS: A novel multiparameter mass spectrometry method to determine the intracellular dynamics of azacitidine therapy in vivo. Leukemia 32, 900-910 (2018).

39. C. H. June, M. Sadelain, Chimeric antigen receptor therapy. N. Engl. J. Med. 379, 64-73 (2018).

40. S. Misra, M. H. Shishehbor, E. A. Takahashi, H. D. Aronow, L. P. Brewster, M. C. Bunte, E. S. H. Kim, J. R. Lindner, K. Rich; American Heart Association Council on Peripheral Vascular Disease; Council on Clinical Cardiology; Council on Cardiovascular and Stroke Nursing, Perfusion assessment in critical limb ischemia: Principles for understanding and the development of evidence and evaluation of devices: A scientific statement from the American Heart Association. Circulation 140, e657-e672 (2019).

41. U. Platzbecker, Treatment of MDS. Blood 133, 1096-1107 (2019).

42. M. Mochizuki, E. Guc, A. J. Park, Z. Julier, P. S. Briquez, G. A. Kuhn, R. Muller, M. A. Swartz, J. A. Hubbell, M. M. Martino, Growth factors with enhanced syndecan binding generate tonic signalling and promote tissue healing. Nat Biomed Eng 4, 463-475 (2020).

43. M. E. Fernyhough, J. L. Vierck, G. J. Hausman, P. S. Mir, E. K. Okine, M. V. Dodson, Primary adipocyte culture: Adipocyte purification methods may lead to a new understanding of adipose tissue growth and development. Cytotechnology 46, 163-172 (2004).

44. H. H. Zhang, S. Kumar, A. H. Barnett, M. C. Eggo, Ceiling culture of mature human adipocytes: Use in studies of adipocyte functions. J. Endocrinol. 164, 119-128 (2000).

45. A. Medvinsky, S. Taoudi, S. Mendes, E. Dzierzak, Analysis and manipulation of hematopoietic progenitor and stem cells from murine embryonic tissues, in Curr Protoc Stem Cell Biol (ed. 2008/09/05, 2008), vol. Chapter 2, pp. Unit 2A 6.

46. M. Marti, L. Mulero, C. Pardo, C. Morera, M. Carrio, L. Laricchia-Robbio, C. R. Esteban, J. C. Izpisua Belmonte, Characterization of pluripotent stem cells. Nat. Protoc. 8, 223-253 (2013).

47. O. Guardiola, G. Andolfi, M. Tirone, F. lavarone, S. Brunelli, G. Minchiotti, Induction of acute skeletal muscle regeneration by cardiotoxin injection. J. Vis. Exp. 2017, 54515 (2017).

48. C. A. Schneider, W. S. Rasband, K. W. Eliceiri, NIH Image to ImageJ: 25 years of image analysis. Nat. Methods 9, 671-675 (2012).

49. S. Chan, S. I. Head, Age- and gender-related changes in contractile properties of non-atrophied EDL muscle. PLoS One 5, e12345 (2010).

50. S. Anders, P. T. Pyl, W. Huber, HTSeq-A Python framework to work with high-throughput sequencing data. Bioinformatics 31, 166-169 (2015).
51. D. Karolchik, A. S. Hinrichs, T. S. Furey, K. M. Roskin, C. W. Sugnet, D. Haussler, W. J. Kent, The UCSC Table Browser data retrieval tool. Nucleic Acids Res. 32, D493-D4496 (2004).

52. M. I. Love, W. Huber, S. Anders, Moderated estimation of fold change and dispersion for RNA-seq data with DESeq2. Genome Biol. 15, 550 (2014).

53. E. Diffner, D. Beck, E. Gudgin, J. A. Thoms, K. Knezevic, C. Pridans, S. Foster, D. Goode, W. K. Lim, L. Boelen, K. H. Metzeler, G. Micklem, S. K. Bohlander, C. Buske, A. Burnett, K. Ottersbach, G. S. Vassiliou, J. Olivier, J. W. Wong, B. Gottgens, B. J. Huntly, J. E. Pimanda, Activity of a heptad of transcription factors is associated with stem cell programs and clinical outcome in acute myeloid leukemia. Blood 121, 2289-2300 (2013).

54. H. Li, R. Durbin, Fast and accurate short read alignment with Burrows-Wheeler transform. Bioinformatics 25, 1754-1760 (2009).

55. Y. Zhang, T. Liu, C. A. Meyer, J. Eeckhoute, D. S. Johnson, B. E. Bernstein, C. Nusbaum, R. M. Myers, M. Brown, W. Li, X. S. Liu, Model-based analysis of ChIP-Seq (MACS). Genome Biol. 9, R137 (2008).

56. G. Yu, L. G. Wang, Q. Y. He, ChIPseeker: An R/Bioconductor package for ChIP peak annotation, comparison and visualization. Bioinformatics 31, 2382-2383 (2015).

57. F. Krueger, S. R. Andrews, Bismark: A flexible aligner and methylation caller for BisulfiteSeq applications. Bioinformatics 27, 1571-1572 (2011).

58. A. Akalin, M. Kormaksson, S. Li, F. E. Garrett-Bakelman, M. E. Figueroa, A. Melnick, C. E. Mason, methylKit: A comprehensive R package for the analysis of genome-wide DNA methylation profiles. Genome Biol. 13, R87 (2012)

59. S. Li, F. E. Garrett-Bakelman, A. Akalin, P. Zumbo, R. Levine, B. L. To, I. D. Lewis, A. L. Brown, R. J. D'Andrea, A. Melnick, C. E. Mason, An optimized algorithm for detecting and annotating regional differential methylation. BMC Bioinformatics 14 (Suppl 5), S10 (2013).

Acknowledgments: We are indebted to the patients who donated tissue to this project. We thank E. Cook (Prince of Wales Private Hospital), B. Lee (Mark Wainwright Analytical Centre, UNSW Sydney), and technicians at the UNSW BRC Facility for assistance with sample and data collection and animal care; Y. Huang for technical assistance; and A. Unnikrishnan and C. Jolly for helpful discussions and critical reading of the manuscript. We acknowledge the facilities and scientific and technical assistance of the National Imaging Facility, a National Collaborative Research Infrastructure Strategy (NCRIS) capability, at the BRIL (UNSW). The STRO-1 antibody was a gift from S. Gronthos, University of Adelaide, Australia. Funding: We acknowledge the following funding support: A.Y. was supported by an Endeavour International Postgraduate Research scholarship from the Australian Government. S.S. is supported by an International Postgraduate Student scholarship from UNSW and the Prince of Wales Clinical School. P.S. is supported by an International Postgraduate Student scholarship from UNSW. M.L.T. and D.D.M. acknowledge funding from St. Vincent's Clinic Foundation and Arrow BMT Foundation. K.A.K. acknowledges funding from Australian Research Council (FT180100417). J.M. is supported, in part, by the Olivia Lambert Foundation. M.K. is supported by a NHMRC Program Grant (APP1091261) and NHMRC Principal Research Fellowship (APP1119152). L.B.H. acknowledges funding from MTPConnect MedTech and Pharma Growth Centre (PRJ2017-55 and BMTH06) as part of the Australian Government-funded Industry Growth Centres Initiative Programme and The Kinghorn Foundation. D.B. is supported by a Peter Doherty Fellowship from the National Health and Medical Research Council of Australia, a Cancer Institute NSW Early Career Fellowship, the Anthony Rothe Memorial Trust, and Gilead Sciences. R.M. acknowledges funding from Jasper Medical Innovations (Sydney, Australia). J.E.P., V.C., and E.C.H. acknowledge funding from the National Health and Medical Research Council of Australia (APP1139811). Author contributions: The project was conceived by V.C. and J.E.P., and the study design and experiments were planned by A.Y., V.C., and J.E.P. Most of the experiments and data analyses were performed by A.Y., guided and supervised by V.C. and J.E.P. S.S., R.A.O., C.A.L., D.C., F.Y., M.L.T., P.S., T.H., J.R.P., P.H., W.R.W., and V.C. performed additional experiments and data analyses, with further supervision from R.M., C.P., J.A.I.T., D.C., J.W.H.W., L.B.H., D.B., and E.C.H. Statistical analyses were performed by J.O. R.M., D.D.M., J.M., K.A.K., and M.K. provided critical reagents. The manuscript was written by A.Y., J.A.I.T., V.C., and J.E.P., and reviewed and agreed to by all coauthors. Competing interests: V.C. and J.E.P. are named inventors on a patent "A method of generating cells with multi-lineage potential" (US 9982232, AUS 2013362880). All other authors declare that they have no competing interests. Data and materials availability: All data needed to evaluate the conclusions in the paper are present in the paper and/or the Supplementary Materials. Additional data related to this paper may be requested from the authors.

Submitted 16 June 2020

Accepted 23 November 2020

Published 13 January 2021

$10.1126 /$ sciadv.abd1929

Citation: A. Yeola, S. Subramanian, R. A. Oliver, C. A. Lucas, J. A. I. Thoms, F. Yan, J. Olivier, D. Chacon M. L. Tursky, P. Srivastava, J. R. Potas, T. Hung, C. Power, P. Hardy, D. D. Ma, K. A. Kilian, J. McCarroll, M. Kavallaris, L. B. Hesson, D. Beck, D. J. Curtis, J. W. H. Wong, E. C. Hardeman, W. R. Walsh, R. Mobbs, V. Chandrakanthan, J. E. Pimanda, Induction of muscle-regenerative multipotent stem cells from human adipocytes by PDGF-AB and 5-azacytidine. Sci. Adv. 7, eabd1929 (2021). 